PRECIPITATION RECORDS AND FLOOD-PRODUCING STORMS IN

CHEYENNE, WYOMING

By J. B. Lindner-Lunsford

U.S. Geological Survey

Water-Resources Investigations Report 87-4225

Prepared in cooperation with the

CITY OF CHEYENNE

Cheyenne, Wyoming

1988

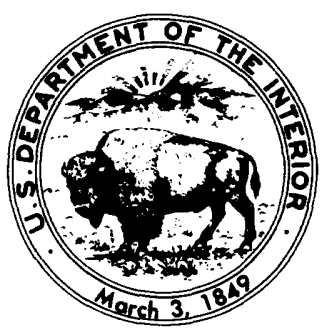




\section{DEPARTMENT OF THE INTERIOR}

DONALD PAUL HODEL, Secretary

U.S. GEOLOGICAL SURVEY

Dallas L. Peck, Director

For additional information write to:

District Chief

U.S. Geological Survey

2120 Capitol Avenue

P.0. Box 1125

Cheyenne, WY 82003
Copies of this report can be purchased from:

U.S. Geological Survey Books and Open-File Reports Section Federal Center, Bldg. 810 Box 25425

Denver, CO 80225 
Abstract ..........................................

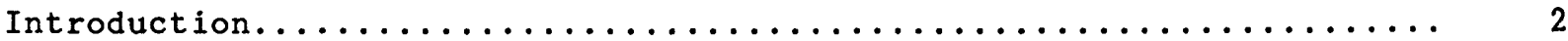

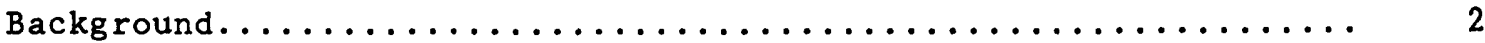

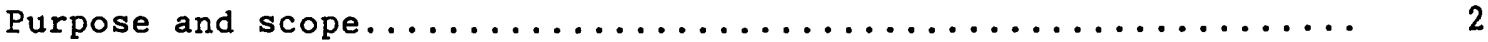

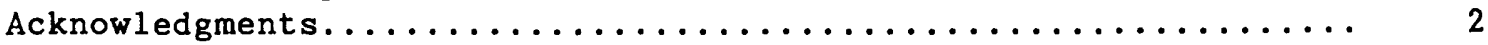

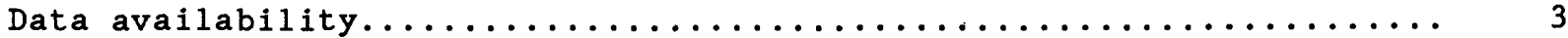

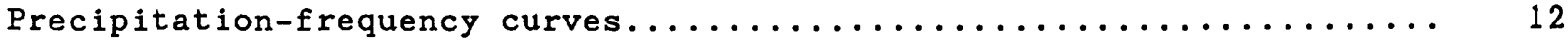

Method of analysis..............................

Results................................... 14

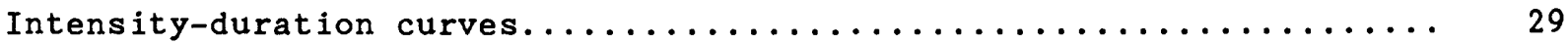

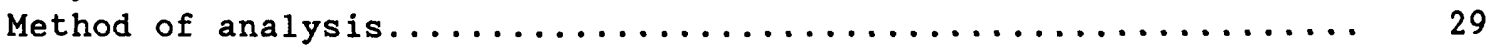

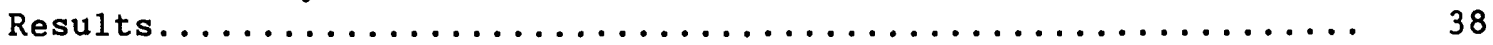

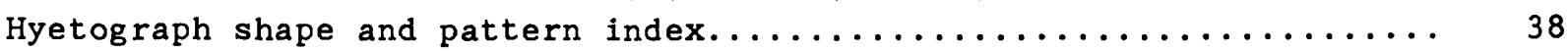

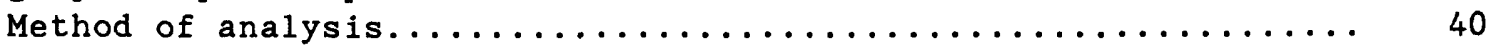

Results......................................41

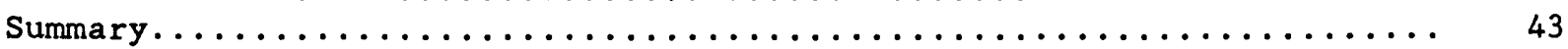

References cited.................................

\section{FIGURES}

Figure 1. Map showing location of study area..............

$2 \mathrm{a}-2 \mathrm{~g}$. Graphs showing precipitation-irequency curves

(arithmetic Gumbe1 distribution) for durations of:

2a. 5 minutes............................... 15

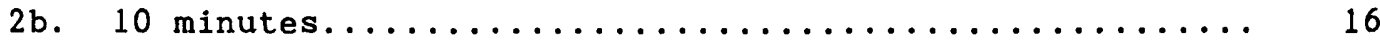

2c. 15 minutes............................ 17

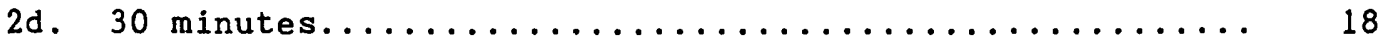

2e. 1 hour............................... 19

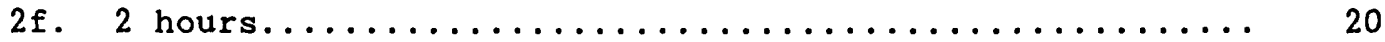

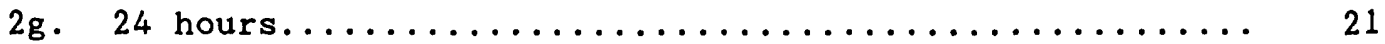

3a-3g. Graphs showing precipitation-frequency curves

(log-Gumbel distribution) for durations of:

3a. 5 minutes.............................. 22

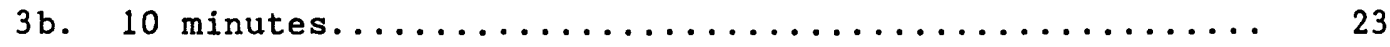

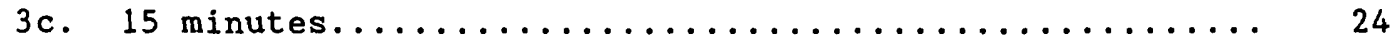

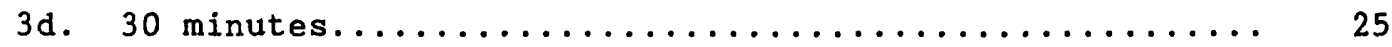

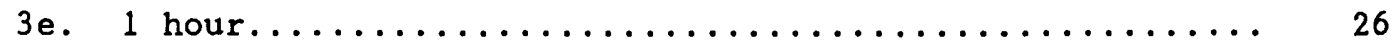

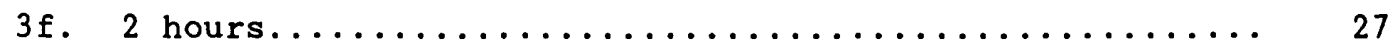

$3 \mathrm{~g} . \quad 24$ hours............................... 28

4a-4g. Graphs showing precipitation-frequency curves

(log-Pearson Type III distribution) for durations of:

4a. 5 minutes.............................. 30

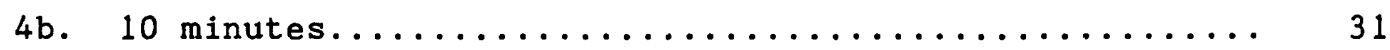

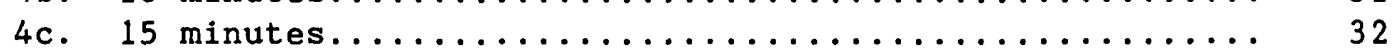

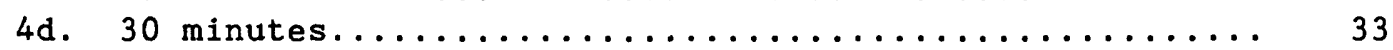

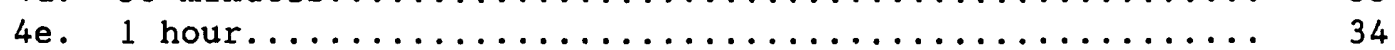

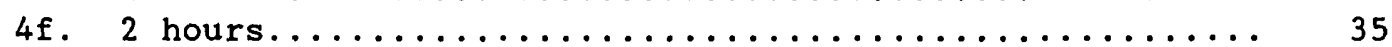

$4 \mathrm{~g} .24$ hours............................. 36 
Figure 5. Graph showing intensity-duration curves for 100-year

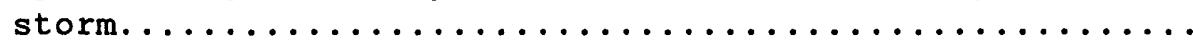

6. Graph showing dimensionless hyetographs for 10

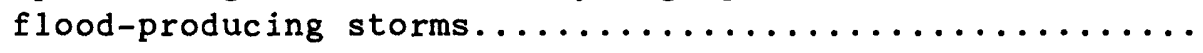

\section{TABLES}

Table 1. Annual maximum precipitation for selected durations,

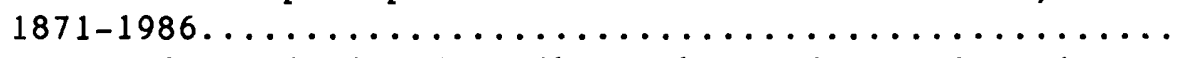

2. Summary of statistics describing observed annual maximum precipitation values for selected durations........... 13

3. Intensity-duration data for 100 -year storm............. 39

4. Precipitation intensity for storms of selected recurrence intervals and durations, using data available through

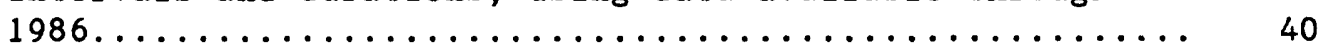

5. Storm characteristics for 10 flood-producing storms....... 41

\section{CONVERSION FACTORS}

For use of readers who prefer to use metric units, rather than the inch-pound units used in this report, the following conversion factors may be used:

\section{Multiply inch-pound unit}

inch

inch per hour
By

25.40

25.40
To obtain metric unit

millimeter millimeter per hour 


\title{
PRECIPITATION RECORDS AND FLOOD-PRODUCING STORMS IN \\ CHEYENNE, WYOMING
}

By J. B. Lindner-Lunsford

\begin{abstract}
Annual maximum precipitation data for Cheyenne, Wyoming are presented for the years 1902-86 for durations of 5, 10, 15, and 30 minutes, and 1 and 2 hours. Annual maximum precipitation data for 24-hour duration are presented for the years 1871-1986. Precipitation-frequency curves are developed using only data collected before 1985. A second set of precipitation frequency curves are developed based on data collected from 1871 through 1986 (including the storm of August 1, 1985). The data are plotted and analyzed in three ways, assuming: (1) The data are described by an arithmetic Gumbel distribution, (2) the logarithms of the data are described by a Gumbel distribution, and (3) the logarithms of the data are described by a Pearson Type III distribution. The inclusion of data for the large storm of August 1, 1985, had the most noticeable effect on the prediction of the magnitude of storms of long recurrence interval for the 1 - and 2-hour durations.

Four different methods of analysis were used to develop seven intensity-duration curves for the 100-year storm. These curves were compared to the observed curve for the August 1, 1985, storm. For durations greater than 30 minutes, the observed curve indicates greater intensity than do five of the seven calculated curves.

Dimensionless hyetographs were developed for 10 flood-producing storms that have occurred in the Cheyenne area since 1903. Pattern index (integral of the dimensionless hyetograph curve) was calculated for each storm. The index for the storm of August 1, 1985, is three standard deviations less than the mean of the pattern indices calculated for the remaining nine storms-indicating that the distribution of precipitation over time for the August 1 storm was outside the normal range for Cheyenne.
\end{abstract}




\section{INTRODUCTION}

\section{$\underline{\text { Background }}$}

A massive thunderstorm that inundated downtown Cheyenne (fig. 1) with up to 7 inches of rain and hail in just over 3 hours (Druse and others, 1986) caused the most devastating flood in Wyoming's history. Twelve deaths, 70 injuries, and $\$ 61.1$ million in damage resulted from the flooding.

As a result of the storm and flood, there is an increased awareness of flood hazards. The City Engineer's Stormwater Advisory Group was formed to assist with suggestions and planning to help alleviate drainage and flooding problems. Recommendations of the Advisory Group include (D. S. Benner, former Cheyenne Assistant City Engineer, written commun., 1986):

1) Designation of the flood plain of the 1985 flood as a no-building area, and implementation of interim planning and building standards until a comprehensive flood-prone area assessment can be performed;

2) Installation of a flood-alert system;

3) Implementation of an urban-hydrology study for development of design information; and

4) Implementation of a comprehensive master-drainage plan and funding program.

The U.S. Geological Survey, in cooperation with the City of Cheyenne, has begun an urban-hydrology study. One phase of this study examines the precipitation record for Cheyenne and evaluates the storm of August 1, 1985, in order to provide background information for the master-drainage plan and urban-hydrology study. The data eventually will be incorporated into an urban rainfall-runoff model for the Cheyenne area.

\section{Purpose and Scope}

This report examines existing precipitation data for Cheyenne and compares three different data distributions to determine which one best describes the Cheyenne data. New precipitation-frequency curves for selected durations are developed for the Cheyenne area on the basis of data collected through 1986. These curves are compared to similar curves developed on the basis of data available before 1985 to determine the influence of the August 1, 1985, storm on predictions of the magnitude of precipitation for various recurrence intervals and durations. Hyetographs for nine other flood-producing storms that occurred in the Cheyenne area between 1904 and 1973 are examined and compared to the hyetograph for the August 1 storm.

\section{Acknowledgments}

The author gratefully acknowledges the assistance of David S. Benner, former Cheyenne Assistant City Engineer, for making helpful comments on pattern index calculations; Brenda Brock, National Weather Service, for assisting in researching old precipitation data; and Felicia A. Reid, U.S. Geological Survey, for writing several preprocessor computer programs used in the analysis of the incremental precipitation data. 


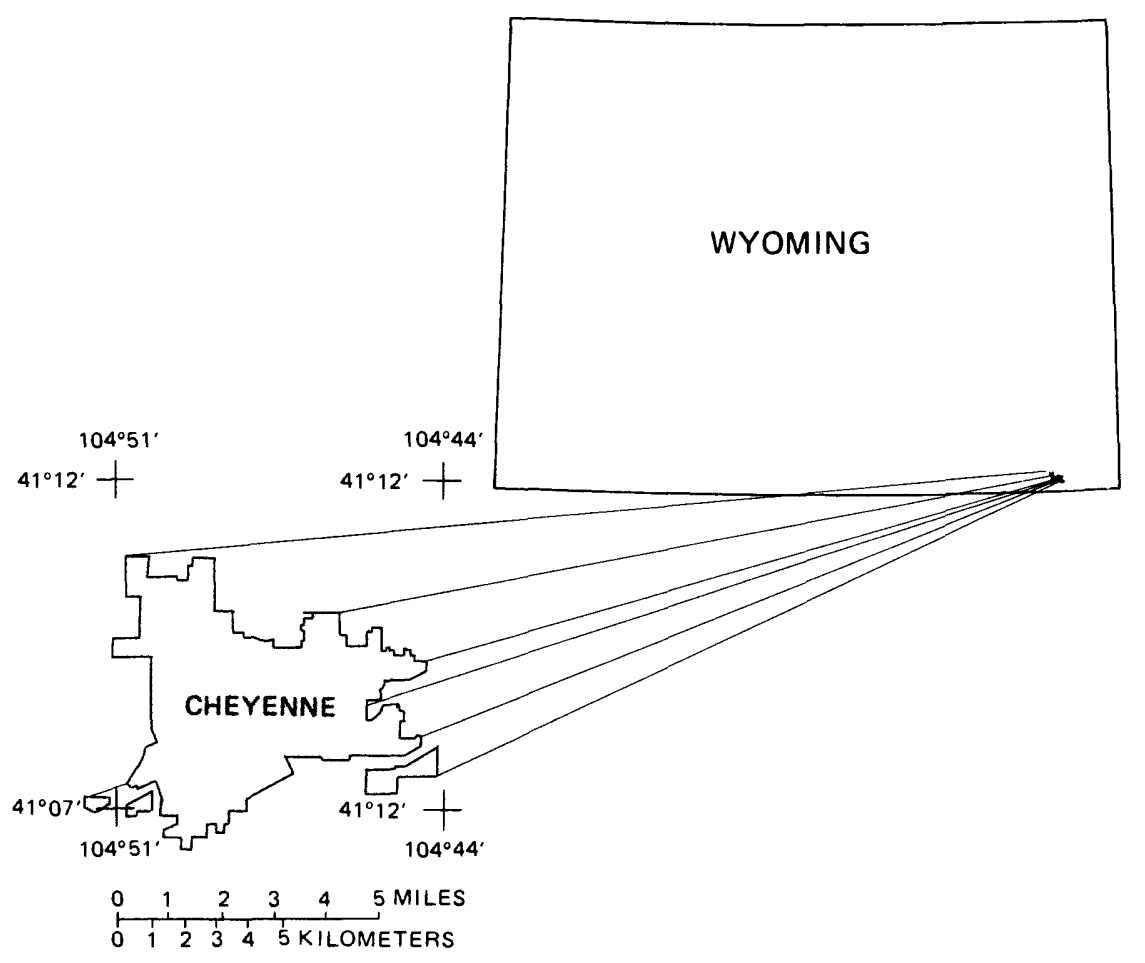

Figure 1.--Location of study area.

\section{DATA AVAILABILITY}

Precipitation data are often used for the purposes of design for flood structures and land-use planning in areas where streamflow records of adequate length are not available. When precipitation data are used for this purpose, the value used will be the maximum amount of precipitation that would occur during a given period, such as 1 or 2 hours, and be exceeded on the average only once in a given number of years, such as 100 years. The maximum amount of precipitation that occurred in any 1-hour period, for example, can be measured and tabulated. Annual maximum 5-, 10-, 15-, and 30-minute and $1-$ and 2-hour-duration precipitation data for Cheyenne for the years 1902-84 (except 1968, 1969, 1971, and 1972) had been compiled previously in computer-readable format by the National Weather Service. Annual maximum 24-hour precipitation was compiled in computerreadable format by the National Weather Service for the period 1891-1984. The author used additional data from the files of the National Weather Service office in Cheyenne to extend the 24-hour record back to 1871 . During the storm and flood of $1896,4.0$ inches of precipitation occurred during a 2-hour period (U.S. Army Corps of Engineers, 1969). This data was added to the 2-hour record. Annual maximum precipitation data for all durations used in this study are published in "Local Climatological Data" monthly summaries (National Oceanic and Atmospheric Administration, 1985 and 1986); these data were added to the record for the period 1985-86. Annual maximum precipitation data used in this report are presented in table 1. 
A record with missing data can be analyzed as though it were a continuous record with a length equal to the number of years of data available if data are missing from the record for reasons not connected with unusual hydrologic occurrences (Interagency Advisory Committee on Water Data, 1982, p. 15). The Cheyenne record has no data for durations less than 1 hour for the years 1968-69 and 1971-72. To determine whether the data were missing due to unusual hydrologic occurrences, the 24-hour record was examined. The 24-hour annual maximum precipitation values for the years 1971-72 were among the smallest values in the record, but the smaller of the two was only 43 percent smaller than the median value in the record. The 24-hour annual maximum precipitation for 1968 was 10 percent larger than the median value

Table 1.--Annual maximum precipitation

[Data from National Weather Service, except

\begin{tabular}{|c|c|c|c|c|c|c|}
\hline \multirow[b]{2}{*}{ Year } & 5-minute & duration & \multicolumn{2}{|c|}{ 10-minute duration } & \multicolumn{2}{|c|}{ 15-minute durat } \\
\hline & $\begin{array}{l}\text { Month } \\
\text { and day }\end{array}$ & $\begin{array}{l}\text { Maximum } \\
\text { rainfa } 11 \\
\text { (inches) }\end{array}$ & $\begin{array}{l}\text { Month } \\
\text { and day }\end{array}$ & $\begin{array}{l}\text { Maximum } \\
\text { rainfal } 1 \\
\text { (inches) }\end{array}$ & $\begin{array}{l}\text { Month } \\
\text { and day }\end{array}$ & $\begin{array}{l}\text { Maximum } \\
\text { rainfal } \\
\text { (inches }\end{array}$ \\
\hline 1871 & ---- & ---- & ----- & ---- & ---- & ---- \\
\hline 1872 & ----- & ---- & ----- & ---- & ----- & ---- \\
\hline 1873 & ----- & ---- & ----- & ---- & ----- & ---- \\
\hline 1874 & ----- & ---- & ----- & ---- & ----- & ---- \\
\hline 1875 & ----- & ---- & ----- & ---- & ----- & ---- \\
\hline 1876 & ---- & ---- & ----- & ---- & ----- & ---- \\
\hline 1877 & ----- & ---- & ----- & ---- & ----- & ---- \\
\hline 1878 & ----- & ---- & ----- & ---- & ----- & ---- \\
\hline 1879 & ----- & ---- & ----- & ---- & ----- & ---- \\
\hline 1880 & ---ー- & ---- & -ーーー- & ---- & ----- & ---- \\
\hline 1881 & ----- & ---- & ----- & ---- & ----- & ---- \\
\hline 1882 & ----- & ---- & ----- & ---- & ----- & ---- \\
\hline 1883 & ----- & ---- & ----- & ---- & ----- & ---- \\
\hline 1884 & ----- & ---- & --ー- & ---- & ----- & ---- \\
\hline 1885 & ----- & ---- & ----- & ---- & ----- & ---- \\
\hline 1886 & ----- & ---- & ----- & ---- & ---- & ---- \\
\hline 1887 & ----- & ---- & ----- & ---- & ----- & ---- \\
\hline 1888 & ----- & ---- & -ー-ー- & ---- & ----- & ---- \\
\hline 1889 & ---ー- & ---- & ----- & ---- & ----- & ---- \\
\hline 1890 & ----- & ---- & ----- & ---- & ---- & ---- \\
\hline 1891 & ----- & ---- & -ー-ー- & ---- & ----- & ---- \\
\hline 1892 & ----- & ---- & ----- & ---- & ----- & ---- \\
\hline 1893 & ----- & ---- & ----- & ---- & ----- & ---- \\
\hline 1894 & ----- & ---- & ----- & ---- & ----- & ---- \\
\hline 1895 & ----- & ---- & -ー--- & ---- & -ー-ー- & ---- \\
\hline
\end{tabular}


in the record. The 1969 value was 28 percent smaller than the median value. These deviations from the median were not extreme, indicating that the years that had missing data for the shorter durations were not anomalous regarding rainfall. To further verify that the inclusion or exclusion of the 4 years of data did not have any significant impact on the record, summary statistics were calculated for the 24-hour record including data for the years 1968-69 and 1971-72 and were compared to the statistics calculated when data for the 4 years were excluded from the record. The mean annual maximum rainfall for the record including the 4 years was 1.547 inches; the mean was 1.565 inches with the 4 years excluded. Standard deviation was 0.753 compared to 0.757 , and skewness was 2.921 compared to 2.916 . Therefore, the data were analyzed as described previously.

for selected durations, 1871-1986

as noted. Dashes indicate missing data]

\section{0-minute duration}

Maximum

Month rainfall

and day (inches)

\section{1-hour duration}

Maximum

Month rainfall and day (inches) 2-hour duration

Maximum

Month rainfall and day (inches) 24-hour duration

$\begin{array}{cl}\text { Month } & \text { Maximum } \\ \text { rainfall } \\ \text { and day } & \text { (inches) }\end{array}$

\begin{tabular}{|c|c|c|c|c|c|c|c|}
\hline---- & ---- & ---- & ---- & ---- & ---- & $5-13$ & 1.54 \\
\hline---- & --- & --- & --- & ---- & --- & $7-25$ & 1.42 \\
\hline - - - & --- & ---- & ---- & ---- & $-\cdots$ & $6-08$ & 1.04 \\
\hline---- & --- & ---- & --- & --- & --- & $10-05$ & 1.35 \\
\hline---- & ---- & $-\cdots$ & --- & --- & --- & $7-24$ & 1.14 \\
\hline --- & --- & ---- & ---- & ---- & ---- & $5-22$ & 1.89 \\
\hline---- & ---- & --- & ---- & ---- & ---- & $9-02$ & 1.07 \\
\hline--- & --- & ---- & $--\cdots$ & ---- & ---- & $5-08$ & 2.47 \\
\hline---- & ---- & ---- & --- & ---- & --- & $5-11$ & 1.15 \\
\hline---- & ---- & ---- & ---- & ---- & ---- & $8-29$ & 1.30 \\
\hline---- & ---- & ---- & --- & ---- & ---- & $4-20$ & 1.23 \\
\hline--- & --- & --- & $\cdots-$ & --- & $-\cdots$ & $7-28$ & .71 \\
\hline$-\cdots-$ & --- & --- & --- & --- & $-\cdots$ & $6-17$ & 1.33 \\
\hline---- & --- & ---- & --- & ---- & --- & $9-15$ & 1.01 \\
\hline---- & ---- & ---- & ---- & ---- & ---- & $4-29$ & 1.33 \\
\hline----- & ---- & ---- & ---- & ---- & ---- & $8-17$ & .56 \\
\hline-- & --- & ---- & --- & ---- & --- & $7-11$ & .84 \\
\hline---- & ---- & ---- & --- & ---- & --- & $9-01$ & 1.15 \\
\hline---- & ---- & ---- & ---- & ---- & --- & $5-10$ & 1.45 \\
\hline ---- & ---- & ---- & ---- & ---- & --- & $7-21$ & 1.45 \\
\hline---- & ---- & ---- & ---- & ---- & $-\cdots$ & $5-16$ & 2.24 \\
\hline-- & --- & ---- & ---- & ---- & ---- & $6-04$ & 1.40 \\
\hline-- & --- & $6-14$ & 0.87 & $\cdots$ & $\cdots$ & $6-14$ & .87 \\
\hline--- & $-\cdots$ & $-\cdots$ & --- & --- & --- & $7-04$ & 1.77 \\
\hline---- & --- & ---- & --- & ---- & --- & $5-31$ & 1.21 \\
\hline
\end{tabular}


Table 1.--Annual maximum precipitation

\begin{tabular}{|c|c|c|c|c|c|c|}
\hline \multirow[b]{2}{*}{ Year } & \multicolumn{2}{|c|}{ 5-minute duration } & \multicolumn{2}{|c|}{ 10-minute duration } & \multicolumn{2}{|c|}{ 15-minute duration } \\
\hline & $\begin{array}{l}\text { Month } \\
\text { and day }\end{array}$ & $\begin{array}{l}\text { Maximum } \\
\text { rainfall } \\
\text { (inches) }\end{array}$ & $\begin{array}{l}\text { Month } \\
\text { and day }\end{array}$ & $\begin{array}{l}\text { Maximum } \\
\text { rainfall } \\
\text { (inches) }\end{array}$ & $\begin{array}{l}\text { Month } \\
\text { and day }\end{array}$ & $\begin{array}{l}\text { Maximum } \\
\text { rainfall } \\
\text { (inches) }\end{array}$ \\
\hline 1896 & --- & ---- & ---- & ---- & ---- & --- \\
\hline 1897 & ---- & ---- & ---- & ---- & ---- & ---- \\
\hline 1898 & $-\ldots-$ & ---- & ----- & ---- & ---- & ---- \\
\hline 1899 & ---- & ---- & ----- & ---- & ---- & --- \\
\hline 1900 & ---- & ---- & ----- & ---- & ---- & ---- \\
\hline 1901 & $9-03$ & 0.18 & $7-26$ & 0.29 & ---- & ---- \\
\hline 1902 & $6-27$ & .18 & $6-27$ & .25 & ----- & ---- \\
\hline 1903 & $8-05$ & .18 & $8-05$ & .26 & $8-05$ & 0.36 \\
\hline 1904 & $5-20$ & .35 & $5-20$ & .57 & $5-20$ & .66 \\
\hline 1905 & $8-30$ & .48 & $7-29$ & .75 & $7-29$ & .94 \\
\hline 1906 & $6-02$ & .25 & $6-02$ & .31 & $7-14$ & .35 \\
\hline 1907 & $7-31$ & .31 & $7-31$ & .44 & $7-31$ & .47 \\
\hline 1908 & $6-02$ & .46 & $6-02$ & .66 & $6-02$ & .84 \\
\hline 1909 & $6-07$ & .42 & $6-07$ & .61 & $6-07$ & .63 \\
\hline 1910 & $7-30$ & .17 & $7-30$ & .25 & $7-30$ & .29 \\
\hline 1911 & $6-15$ & .28 & $8-09$ & .41 & $8-09$ & .46 \\
\hline 1912 & $8-18$ & .25 & $8-18$ & .40 & $8-18$ & .44 \\
\hline 1913 & $9-02$ & .41 & $9-02$ & .64 & $9-02$ & .69 \\
\hline 1914 & $8-25$ & .25 & $8-25$ & .50 & $8-25$ & .58 \\
\hline 1915 & $8-23$ & .38 & $8-23$ & .62 & $8-23$ & .70 \\
\hline 1916 & $7-29$ & .27 & $8-15$ & .44 & $8-15$ & .58 \\
\hline 1917 & $8-19$ & .18 & $7-10$ & .29 & $8-19$ & .41 \\
\hline 1918 & $8-14$ & .30 & $8-14$ & .50 & $8-14$ & .65 \\
\hline 1919 & $7-30$ & .18 & $7-14$ & .29 & $7-14$ & .36 \\
\hline 1920 & $9-06$ & .20 & $9-06$ & .38 & $9-06$ & .51 \\
\hline 1921 & $5-06$ & .06 & $5-06$ & .09 & $5-06$ & .13 \\
\hline 1922 & $7-20$ & .35 & $7-20$ & .54 & $7-20$ & .56 \\
\hline 1923 & $7-13$ & .32 & $7-13$ & .55 & $7-13$ & .82 \\
\hline 1924 & $7-30$ & .41 & $7-30$ & .56 & $7-30$ & .62 \\
\hline 1925 & $7-27$ & .36 & $7-27$ & .57 & $7-27$ & .70 \\
\hline 1926 & $6-14$ & .49 & $6-14$ & .93 & $6-14$ & 1.36 \\
\hline 1927 & $8-15$ & .30 & $8-15$ & .35 & $8-15$ & .37 \\
\hline 1928 & $7-18$ & .30 & $7-18$ & .49 & $7-18$ & .63 \\
\hline 1929 & $8-20$ & .29 & $8-20$ & .35 & $8-20$ & .53 \\
\hline 1930 & $8-18$ & .40 & $8-18$ & .69 & $8-18$ & .86 \\
\hline
\end{tabular}


for selected durations, 1871-1986--Continued

30-minute duration

Maximum
Month rainfall

and day (inches)

\section{1-hour duration}

Maximum

Month rainfall and day (inches) 2-hour duration

Maximum

Month rainfall and day (inches) 24-hour duration

Maximum

Month rainfall

and day (inches)

\begin{tabular}{|c|c|c|c|c|c|c|c|}
\hline---- & --- & $-\ldots$ & --- & $7-15$ & 4.0 & $7-15$ & 4.7 \\
\hline--- & --- & --- & --- & $\ldots-$ & ---- & $7-09$ & 1.75 \\
\hline---- & --- & $-\ldots$ & ---- & ---- & ---- & $6-11$ & 1.18 \\
\hline---- & --- & $-\cdots$ & $-\cdots$ & --- & --- & $5-20$ & 1.18 \\
\hline$-\cdots-$ & --- & $-\ldots$ & $-\cdots$ & --- & --- & $4-05$ & 1.55 \\
\hline---- & --- & $7-26$ & 0.65 & $\ldots$ & ---- & $6-15$ & 1.19 \\
\hline---- & --- & $5-08$ & 1.24 & ---- & --- & $9-22$ & 2.87 \\
\hline $8-05$ & 0.50 & $8-05$ & .50 & $8-05$ & .50 & $4-12$ & 1.24 \\
\hline $5-20$ & .82 & $5-20$ & .97 & $5-20$ & 1.08 & $5-03$ & 2.71 \\
\hline $8-30$ & 1.20 & $8-30$ & 1.29 & $8-30$ & 1.29 & $4-20$ & 2.15 \\
\hline $7-14$ & .41 & $7-14$ & .51 & $7-14$ & .58 & $4-07$ & 1.33 \\
\hline $7-25$ & .50 & $7-25$ & .57 & $7-25$ & .65 & $5-13$ & 1.18 \\
\hline $6-02$ & .89 & $6-02$ & 1.03 & $6-02$ & 1.16 & $5-24$ & 2.67 \\
\hline $6-07$ & .65 & $6-07$ & .74 & $6-07$ & .77 & $6-08$ & 1.15 \\
\hline $7-30$ & .30 & $7-16$ & .32 & $9-20$ & .57 & $3-29$ & 1.43 \\
\hline $8-09$ & .47 & $6-15$ & .47 & $9-30$ & .66 & $10-01$ & 1.45 \\
\hline $8-18$ & .59 & $8-18$ & .61 & $9-12$ & .64 & $9-10$ & 1.56 \\
\hline $9-02$ & .93 & $9-02$ & .94 & $9-02$ & .94 & $9-02$ & .97 \\
\hline $8-25$ & .69 & $8-25$ & .75 & $8-25$ & .75 & $8-26$ & .94 \\
\hline $8-23$ & .98 & $8-23$ & 1.18 & $8-23$ & 1.54 & $8-23$ & 1.72 \\
\hline $8-15$ & .60 & $8-15$ & .64 & $7-29$ & .89 & $5-20$ & 1.16 \\
\hline $8-19$ & .58 & $8-19$ & .61 & $8-19$ & .65 & $5-25$ & 1.70 \\
\hline $8-14$ & .76 & $7-18$ & .81 & $7-18$ & .83 & $7-09$ & 1.11 \\
\hline $7-14$ & .38 & $7-14$ & .48 & $7-14$ & .62 & $7-31$ & .93 \\
\hline $9-06$ & .86 & $9-06$ & 1.36 & $9-06$ & 1.57 & $9-06$ & 1.59 \\
\hline $6-03$ & .20 & $6-03$ & .34 & $6-03$ & .52 & $1-24$ & 1.17 \\
\hline $7-20$ & .58 & $7-20$ & .70 & $7-20$ & .71 & $11-05$ & 1.32 \\
\hline $7-13$ & 1.01 & $9-27$ & 1.40 & $9-27$ & 1.53 & $4-22$ & 2.65 \\
\hline $6-08$ & .74 & $6-08$ & .93 & $6-08$ & .93 & $5-27$ & 1.55 \\
\hline $7-27$ & 1.06 & $7-27$ & 1.31 & $7-27$ & 1.31 & $7-28$ & 1.37 \\
\hline $6-14$ & 2.08 & $6-14$ & 2.51 & $6-14$ & 2.54 & $6-14$ & 3.24 \\
\hline $8-14$ & .58 & $8-14$ & .95 & $8-14$ & 1.18 & $8-15$ & 1.60 \\
\hline $7-18$ & .69 & $7-18$ & .70 & $7-18$ & .71 & $6-03$ & 1.05 \\
\hline $8-20$ & .67 & $8-20$ & .68 & $8-20$ & .68 & $4-24$ & 3.20 \\
\hline $8-18$ & .89 & $8-18$ & .90 & $8-18$ & .91 & $5-17$ & 1.48 \\
\hline
\end{tabular}


Table 1.--Annual maximum precipitation

\begin{tabular}{|c|c|c|c|c|c|c|}
\hline \multirow[b]{2}{*}{ Year } & \multicolumn{2}{|c|}{ 5-minute duration } & \multicolumn{2}{|c|}{ 10-minute duration } & \multicolumn{2}{|c|}{ 15-minute duration } \\
\hline & $\begin{array}{l}\text { Month } \\
\text { and day }\end{array}$ & $\begin{array}{l}\text { Maximum } \\
\text { rainfa } 11 \\
\text { (inches) }\end{array}$ & $\begin{array}{l}\text { Month } \\
\text { and day }\end{array}$ & $\begin{array}{l}\text { Maximum } \\
\text { rainfall } \\
\text { (inches) }\end{array}$ & $\begin{array}{l}\text { Month } \\
\text { and day }\end{array}$ & $\begin{array}{l}\text { Maximum } \\
\text { rainfal1 } \\
\text { (inches) }\end{array}$ \\
\hline 1931 & $8-19$ & 0.28 & $8-19$ & 0.50 & $8-19$ & 0.62 \\
\hline 1932 & $6-09$ & .20 & $6-09$ & .32 & $9-24$ & .40 \\
\hline 1933 & $6-19$ & .22 & $6-19$ & .35 & $6-19$ & .42 \\
\hline 1934 & $7-26$ & .39 & $7-26$ & .74 & $7-26$ & 1.06 \\
\hline 1935 & $6-10$ & .59 & $6-10$ & .93 & $6-10$ & 1.22 \\
\hline 1936 & $9-03$ & .52 & $9-03$ & .66 & $7-20$ & .70 \\
\hline 1937 & $8-12$ & .34 & $8-12$ & .38 & $8-12$ & .41 \\
\hline 1938 & $8-15$ & .29 & $8-15$ & .44 & $8-15$ & .46 \\
\hline 1939 & $6-28$ & .16 & $6-28$ & .22 & $5-06$ & .28 \\
\hline 1940 & $7-02$ & .33 & $7-02$ & .52 & $7-02$ & .61 \\
\hline 1941 & $7-11$ & .21 & $7-11$ & .30 & $10-21$ & .33 \\
\hline 1942 & $8-03$ & .29 & $8-03$ & .48 & $8-03$ & .57 \\
\hline 1943 & $7-27$ & .19 & $7-27$ & .20 & $6-26$ & .30 \\
\hline 1944 & $7-10$ & .45 & $7-10$ & .70 & $7-10$ & 1.01 \\
\hline 1945 & $8-01$ & .23 & $8-01$ & .31 & $8-01$ & .42 \\
\hline 1946 & $8-26$ & .66 & $8-26$ & .98 & $8-26$ & 1.06 \\
\hline 1947 & $6-20$ & .26 & $6-20$ & .44 & $7-20$ & .71 \\
\hline 1948 & $6-27$ & .29 & $6-27$ & .31 & $6-27$ & .43 \\
\hline 1949 & $7-14$ & .18 & $7-14$ & .31 & $7-14$ & .46 \\
\hline 1950 & $7-21$ & .25 & $7-21$ & .29 & $7-21$ & .32 \\
\hline 1951 & $9-06$ & .29 & $9-06$ & .43 & $5-15$ & .65 \\
\hline 1952 & $8-04$ & .33 & $8-04$ & .42 & $8-03$ & .44 \\
\hline 1953 & $7-15$ & .29 & $7-15$ & .52 & $6-06$ & .73 \\
\hline 1954 & $6-14$ & .20 & $6-14$ & .36 & $6-14$ & .43 \\
\hline 1955 & $6-21$ & .33 & $6-21$ & .52 & $6-21$ & .59 \\
\hline 1956 & $5-28$ & .18 & $5-28$ & .29 & $5-28$ & .41 \\
\hline 1957 & $5-29$ & .38 & $7-18$ & .50 & $7-18$ & .58 \\
\hline 1958 & $7-24$ & .40 & $7-24$ & .58 & $7-24$ & .76 \\
\hline 1959 & $8-13$ & .26 & $8-13$ & .42 & $8-13$ & .44 \\
\hline 1960 & $6-11$ & .24 & $7-04$ & .33 & $7-04$ & .36 \\
\hline 1961 & $8-16$ & .44 & $8-16$ & .55 & $8-16$ & .65 \\
\hline 1962 & $7-01$ & .30 & $7-01$ & .53 & $7-01$ & .63 \\
\hline 1963 & $9-08$ & .30 & $9-08$ & .42 & $9-08$ & .65 \\
\hline 1964 & $7-25$ & .21 & $7-25$ & .31 & $7-25$ & .37 \\
\hline 1965 & $8-03$ & .40 & $8-03$ & .60 & $8-03$ & .77 \\
\hline
\end{tabular}




\begin{tabular}{|c|c|c|c|c|c|c|c|}
\hline \multicolumn{2}{|c|}{ 30-minute duration } & \multicolumn{2}{|c|}{ 1-hour duration } & \multicolumn{2}{|c|}{ 2-hour duration } & \multicolumn{2}{|c|}{ 24-hour duration } \\
\hline $\begin{array}{l}\text { Month } \\
\text { and day }\end{array}$ & $\begin{array}{l}\text { Maximum } \\
\text { rainfal1 } \\
\text { (inches) }\end{array}$ & $\begin{array}{l}\text { Month } \\
\text { and day }\end{array}$ & $\begin{array}{l}\text { Maximum } \\
\text { rainfal1 } \\
\text { (inches) }\end{array}$ & $\begin{array}{l}\text { Month } \\
\text { and day }\end{array}$ & $\begin{array}{l}\text { Maximum } \\
\text { rainfall } \\
\text { (inches) }\end{array}$ & $\begin{array}{l}\text { Month } \\
\text { and day }\end{array}$ & $\begin{array}{l}\text { Maximum } \\
\text { rainfal } \\
\text { (inches) }\end{array}$ \\
\hline
\end{tabular}

\begin{tabular}{|c|c|c|c|c|c|c|c|}
\hline $8-19$ & 0.82 & $8-19$ & 0.93 & $8-15$ & 0.94 & $5-29$ & 1.11 \\
\hline $9-24$ & .67 & $9-24$ & .95 & $9-24$ & 1.02 & $9-24$ & 1.09 \\
\hline $6-19$ & .47 & $6-19$ & .48 & $6-19$ & .49 & $4-20$ & 1.66 \\
\hline $7-26$ & 1.33 & $7-26$ & 1.36 & $7-26$ & 1.36 & $7-26$ & 1.36 \\
\hline $6-10$ & 1.30 & $6-10$ & 1.30 & $6-10$ & 1.30 & $5-18$ & 1.76 \\
\hline $7-20$ & .89 & $7-20$ & .90 & $9-03$ & .91 & $6-10$ & 1.55 \\
\hline $7-15$ & .52 & $7-15$ & .52 & $7-15$ & .53 & $3-19$ & .81 \\
\hline $6-21$ & .47 & $6-21$ & .55 & $6-21$ & .59 & $9-02$ & 1.10 \\
\hline $5-06$ & .35 & $5-06$ & .40 & $5-06$ & .40 & $1-09$ & .53 \\
\hline $7-02$ & .81 & $7-02$ & .87 & $7-02$ & .93 & $9-20$ & 1.32 \\
\hline $10-21$ & .40 & $6-07$ & .62 & $6-07$ & .95 & $6-08$ & 2.17 \\
\hline $8-03$ & .60 & $8-03$ & .60 & $8-03$ & .60 & $5-02$ & 2.00 \\
\hline $6-26$ & .47 & $6-26$ & .54 & $6-26$ & .55 & $5-17$ & 1.16 \\
\hline $7-10$ & 1.22 & $7-10$ & 1.23 & $7-10$ & 1.23 & $7-10$ & 1.70 \\
\hline $8-01$ & .45 & $6-06$ & .46 & $6-06$ & .58 & $6-15$ & 1.02 \\
\hline $8-26$ & 1.31 & $8-26$ & 1.45 & $8-26$ & 1.45 & $3-15$ & 1.88 \\
\hline $7-20$ & 1.25 & $7-20$ & 1.25 & $7-20$ & 1.26 & $6-21$ & 1.91 \\
\hline $6-27$ & .50 & $6-27$ & .54 & $6-27$ & .87 & $6-27$ & 1.04 \\
\hline $8-14$ & .61 & $8-14$ & .84 & $8-14$ & .94 & $5-21$ & 1.58 \\
\hline $7-21$ & .40 & $7-21$ & .43 & $7-21$ & .43 & $5-25$ & 1.24 \\
\hline $5-15$ & .70 & $5-15$ & .71 & $5-15$ & .74 & $10-05$ & 1.34 \\
\hline $8-03$ & .49 & $8-03$ & .50 & $8-03$ & .59 & $5-16$ & 1.22 \\
\hline $6-06$ & .99 & $6-06$ & 1.00 & $6-06$ & 1.26 & $6-07$ & 1.95 \\
\hline $6-14$ & .44 & $6-14$ & .46 & $6-14$ & .46 & $3-13$ & 1.19 \\
\hline $6-21$ & .65 & $6-14$ & .92 & $6-14$ & 1.54 & $6-15$ & 2.68 \\
\hline $5-28$ & .53 & $5-28$ & .55 & $5-28$ & .56 & $5-27$ & .85 \\
\hline $7-18$ & .64 & $7-18$ & .80 & $7-18$ & .80 & $6-15$ & 1.26 \\
\hline $7-24$ & .84 & $7-24$ & .95 & $7-24$ & 1.05 & $7-24$ & 1.16 \\
\hline $8-13$ & .46 & $8-13$ & .46 & $8-13$ & .46 & $5-22$ & 1.48 \\
\hline $7-04$ & .38 & $6-11$ & .67 & $6-11$ & .76 & $6-11$ & .89 \\
\hline $8-16$ & .84 & $8-16$ & 1.03 & $8-16$ & 1.40 & $8-17$ & 1.96 \\
\hline $7-01$ & .66 & $7-01$ & .77 & $7-01$ & .77 & $9-24$ & .99 \\
\hline $9-08$ & .84 & $9-08$ & 1.01 & $9-08$ & 1.08 & $9-09$ & 1.78 \\
\hline $7-25$ & .57 & $7-25$ & .60 & $7-25$ & .60 & $7-26$ & .68 \\
\hline $8-03$ & .77 & $6-09$ & .80 & $6-09$ & .86 & $6-10$ & 1.45 \\
\hline
\end{tabular}


Table 1.--Annual maximum precipitation

\begin{tabular}{|c|c|c|c|c|c|c|}
\hline \multirow[b]{2}{*}{ Year } & \multicolumn{2}{|c|}{ 5-minute duration } & \multicolumn{2}{|c|}{ 10-minute duration } & \multicolumn{2}{|c|}{ 15-minute duration } \\
\hline & $\begin{array}{l}\text { Month } \\
\text { and day }\end{array}$ & $\begin{array}{l}\text { Maximum } \\
\text { rainfal1 } \\
\text { (inches) }\end{array}$ & $\begin{array}{l}\text { Month } \\
\text { and day }\end{array}$ & $\begin{array}{l}\text { Maximum } \\
\text { rainfall } \\
\text { (inches) }\end{array}$ & $\begin{array}{l}\text { Month } \\
\text { and day }\end{array}$ & $\begin{array}{l}\text { Maximum } \\
\text { rainfal1 } \\
\text { (inches) }\end{array}$ \\
\hline 1966 & $7-22$ & 0.41 & $9-01$ & 0.54 & $9-01$ & 0.65 \\
\hline 1967 & $5-29$ & .13 & $5-29$ & .23 & $5-29$ & .30 \\
\hline 1968 & ---- & $\left({ }^{1}\right)$ & ---- & $\left({ }^{1}\right)$ & ---- & $\left({ }^{1}\right)$ \\
\hline 1969 & ---- & $\left({ }^{1}\right)$ & ---- & $(1)$ & ---- & $(1)$ \\
\hline 1970 & $5-24$ & .44 & $5-24$ & .75 & $5-24$ & .82 \\
\hline 1971 & ---- & $\left({ }^{1}\right)$ & ---- & $\left({ }^{1}\right)$ & ---- & $\left({ }^{1}\right)$ \\
\hline 1972 & ---- & (1) & --- & $\left({ }^{1}\right)$ & ---- & $\left({ }^{1}\right)$ \\
\hline 1973 & $9-08$ & .60 & $9-08$ & 1.00 & $9-08$ & 1.12 \\
\hline 1974 & $8-28$ & .24 & $8-28$ & .39 & $8-28$ & .52 \\
\hline 1975 & $6-18$ & .23 & $6-18$ & .30 & $6-18$ & .33 \\
\hline 1976 & $7-21$ & .20 & $7-21$ & .40 & $7-21$ & .48 \\
\hline 1977 & $6-23$ & .26 & $7-14$ & .42 & $7-14$ & .46 \\
\hline 1978 & $7-21$ & .18 & $7-21$ & .30 & $8-08$ & .35 \\
\hline 1979 & $7-30$ & .40 & $7-30$ & .53 & $7-30$ & .59 \\
\hline 1980 & $7-24$ & .25 & $7-23$ & .30 & $7-23$ & .33 \\
\hline 1981 & $7-12$ & .50 & $7-12$ & .87 & $7-12$ & .89 \\
\hline 1982 & $6-25$ & .26 & $6-25$ & .52 & $5-23$ & .70 \\
\hline 1983 & $8-17$ & .30 & $8-17$ & .60 & $8-17$ & .75 \\
\hline 1984 & $7-24$ & .24 & $7-24$ & .41 & $8-02$ & .46 \\
\hline 1985 & $8-01$ & .87 & $8-01$ & 1.08 & $8-01$ & 1.29 \\
\hline 1986 & 4-07 & .16 & $9-18$ & .25 & $9-18$ & .34 \\
\hline
\end{tabular}

1 The original design of this study included completing periods of 1971-72 by using 5-minute incremental precipitation data collected 5-minute incremental precipitation for every storm during the period greatest quantity of runoff were analyzed. The storm containing the would only have been analyzed by Craig and Rankl if that storm also that occurred that year. Periods of missing data in the record were intense rainfall of the year was represented in the available data.

2 Data from Craig and Rankl (1978). Data is presented for 


\begin{tabular}{|c|c|c|c|c|c|c|c|}
\hline \multicolumn{2}{|c|}{ 30-minute duration } & \multicolumn{2}{|c|}{ 1-hour duration } & \multicolumn{2}{|c|}{ 2-hour duration } & \multicolumn{2}{|c|}{ 24-hour duration } \\
\hline $\begin{array}{l}\text { Month } \\
\text { and day }\end{array}$ & $\begin{array}{l}\text { Maximum } \\
\text { rainfall } \\
\text { (inches) }\end{array}$ & $\begin{array}{l}\text { Month } \\
\text { and day }\end{array}$ & $\begin{array}{l}\text { Maximum } \\
\text { rainfall } \\
\text { (inches) }\end{array}$ & $\begin{array}{l}\text { Month } \\
\text { and day }\end{array}$ & $\begin{array}{l}\text { Maximum } \\
\text { rainfal1 } \\
\text { (inches) }\end{array}$ & $\begin{array}{l}\text { Month } \\
\text { and day }\end{array}$ & $\begin{array}{l}\text { Maximum } \\
\text { rainfal1 } \\
\text { (inches) }\end{array}$ \\
\hline $7-31$ & 0.91 & $7-31$ & 1.16 & $7-31$ & 1.39 & $7-31$ & 1.70 \\
\hline $5-29$ & .46 & $5-29$ & .52 & $5-29$ & .53 & $4-13$ & 1.63 \\
\hline---- & $(1)$ & $27-25$ & 2.50 & $25-23$ & 2.75 & $5-23$ & 1.52 \\
\hline---- & $(1)$ & $10-05$ & 2.35 & $210-05$ & 2.70 & $6-11$ & .98 \\
\hline $5-24$ & .88 & $5-24$ & .96 & $5-24$ & .96 & $6-12$ & 1.87 \\
\hline---- & $\left({ }^{1}\right)$ & $28-28$ & 2.45 & $28-28$ & 2.45 & $5-05$ & .85 \\
\hline---- & $(1)$ & $25-19$ & 2.35 & $25-19$ & 2.45 & $5-19$ & .78 \\
\hline $9-08$ & 2.00 & $9-08$ & 2.48 & $9-08$ & 2.68 & $7-19$ & 3.42 \\
\hline $8-28$ & .73 & $8-28$ & .75 & $8-28$ & .75 & $6-08$ & 2.33 \\
\hline $6-18$ & .42 & $7-14$ & .61 & $7-14$ & 1.01 & $5-29$ & 1.21 \\
\hline $7-21$ & .57 & $7-21$ & .73 & $7-21$ & .73 & $7-21$ & 1.08 \\
\hline $7-24$ & .82 & $7-24$ & .94 & $7-24$ & 1.13 & $7-25$ & 1.67 \\
\hline $8-08$ & .46 & $8-08$ & .57 & $8-08$ & .72 & $5-06$ & 1.50 \\
\hline $7-30$ & .62 & $7-30$ & .65 & $7-30$ & .65 & $11-20$ & 1.65 \\
\hline $7-23$ & .40 & $7-23$ & .45 & $8-15$ & .54 & $1-25$ & 1.18 \\
\hline $7-12$ & .97 & $8-12$ & 1.31 & $8-12$ & 1.40 & $7-12$ & 1.76 \\
\hline $5-23$ & .95 & $5-23$ & 1.28 & $6-25$ & 1.46 & $6-24$ & 1.76 \\
\hline $8-17$ & 1.14 & $8-17$ & 1.20 & $8-17$ & 1.20 & $5-16$ & 1.38 \\
\hline $7-24$ & .55 & $7-24$ & .75 & $7-24$ & .88 & $4-19$ & 1.94 \\
\hline $8-01$ & 2.19 & $8-01$ & 3.64 & $8-01$ & 4.69 & $8-01$ & 6.06 \\
\hline $9-18$ & .64 & $9-18$ & 1.15 & $9-18$ & 1.40 & $9-18$ & 1.40 \\
\hline
\end{tabular}

missing data in the National Weather Service record for the years 1968-69 and by Craig and Rankl (1978). However, Craig and Rankl (1978) did not determine of interest. Instead, only the one to three storms each year that produced the most intense 5-minute period of precipitation that occurred during a given year was one of the one to three storms that produced the greatest amount of runoff not completed as originally planned because there was no assurance that the most

comparison purposes; however, it was not used in the anaylsis. 
Precipitation data for 5-, 10-, 15-, and 30-minute, and 1-, 2-, and 24hour durations were used in this study. Amount of precipitation for each duration is plotted against "recurrence interval"--the length of time in which the given amount of precipitation can be expected to be equalled or exceeded once. The resulting graph is called a precipitation-frequency curve. The analytical procedure is based on the assumption that the data are described by one of several possible data distributions. Three different distributions were examined in this report to determine which one seemed to best fit the Cheyenne data.

\section{Method of Analysis}

Annual maximum precipitation data for 5-, 10-, 15-, and 30-minute, and 1-, 2-, and 24-hour durations were analyzed three times, based on the assumption that: (1) The data were described by a Gumbel distribution, (2) the logarithms of the data were described by a Gumbel distribution (referred to as "log-Gumbel distribution" in this report), or (3) the logarithms of the data were described by a Pearson Type III distribution (referred to as "log-Pearson Type III distribution" in this report). Data for each duration and assumed distribution were examined twice. The first analysis, using only data collected before 1985, created the frequency curves defined before the storm of August 1, 1985, (subsequently referred to as "previously defined curves"). Precipitation-frequency curves were then updated by including all data collected through 1986 in order to assess the effects of the August 1 storm on predicted storm magnitudes.

The initial analysis was carried out assuming that the data were described by a Gumbel distribution because the Gumbel distribution is used by the National Weather Service for analyzing precipitation data. The method used in this report for fitting a straight line to the precipitation data is identical to the method used by the National Weather Service (Frank Richards, National Weather Service, oral commun., 1987, and Robert Lichty, U.S. Geologica1 Survey, written commun., 1987). More1-Seytoux (1986, p. 75) cites a previous study that demonstrated the legitimacy of using the assumption of a Gumbel distribution to extrapolate precipitation data up to a 1,000-year recurrence interval based on 25 years of data. However, Chow (1964, p. 8-35) cites studies that indicate the Gumbel approximation underpredicts the maximum values of rainfall for long recurrence intervals.

The Gumbel distribution is based on only two parameters, the mean and the standard deviation, and assumes a constant skewness of 1.14. Skewness was calculated for all the rainfall durations of interest for all data available through 1986 and ranged from 0.984 to 3.41 (table 2). For a given mean and standard deviation, the higher the skewness, the higher the estimation of the magnitude of the 100-year event will be. Particularly for the 1-, 2-, and 24-hour arithmetic data, the actual value of skewness is substantially higher than the value assumed in the Gumbel distribution. Therefore, a straight line based on the Gumbel distribution fitted to this data may underpredict the magnitude of the 100-year storm. 
Table 2.--Summary of statistics describing observed annual maximum

precipitation values for selected durations

\begin{tabular}{|c|c|c|c|}
\hline Duration & Mean & $\begin{array}{l}\text { Standard } \\
\text { deviation }\end{array}$ & Skewness \\
\hline & \multicolumn{3}{|c|}{ Arithmetic data } \\
\hline 5 minutes & 0.311 & 0.130 & 1.42 \\
\hline 10 minutes & .476 & .198 & .984 \\
\hline 15 minutes & .589 & .245 & 1.06 \\
\hline 30 minutes & .749 & .369 & 1.90 \\
\hline 1 hour & .864 & .494 & 2.91 \\
\hline 2 hours & 1.00 & .668 & 3.41 \\
\hline \multirow[t]{2}{*}{24 hours } & 1.55 & .753 & 2.92 \\
\hline & \multicolumn{3}{|c|}{ Logarithmica11y transformed data } \\
\hline 5 minutes & -.542 & .177 & -.355 \\
\hline 10 minutes & -.359 & .183 & -.401 \\
\hline 15 minutes & -.265 & .179 & -.207 \\
\hline 30 minutes & -.168 & .188 & .303 \\
\hline 1 hour & -.112 & .198 & .595 \\
\hline 2 hours & -.055 & .205 & .995 \\
\hline 24 hours & .153 & .169 & .655 \\
\hline
\end{tabular}

A second analysis was carried out--this time based on the assumption that the logarithms of the data were described by a Gumbel distribution. Again, the method of the National Weather Service was used to fit a straight line to the data. Skewnesses of the logarithmically transformed data ranged from -0.359 to 0.995 . The logarithmically transformed data for durations of 1 hour or less have skewnesses substantially smaller than the skewness of 1.14 assumed in the Gumbel distribution. Therefore, a straight line based on the log-Gumbel distribution fitted to this data may overpredict the magnitude of the 100-year storm.

Because the calculated skewness of both the data and the logarithms of the data for certain durations was substantially different from the value of 1.14 assumed by the Gumbel distribution, another distribution was examined to see if it would better describe the data. The log-Pearson Type III distribution was chosen because the log-Pearson Type III distribution, unlike the Gumbel distribution, is based on three parameters: Mean, standard deviation, and skewness. The analysis utilized Geological Survey computer program J407 (Lepkin and others, 1979). 
The log-Pearson Type III distribution is not recommended for analysis of precipitation data by the National Weather Service. The National Weather Service position is that skewness is too susceptible to influence of sampling error to be a reliable parameter in describing storm data (Frank Richards, National Weather Service, oral commun., 1987); however, the likelihood of sampling error having a significant effect on the calculated value of skewness decreases with the length of the record being considered. There is no theoretical reason (other than the previously described sensitivity of the skewness to sampling error) for not using the log-Pearson Type III distribution. In fact, the State of California Department of Water Resources uses the log-Pearson Type III distribution for analys is of precipitation data, rather than the Gumbel distribution, in order to incorporate the effects of the skewness of the data into their analyses.

\section{Results}

Ranked precipitation data for 5-, 10-, 15-, and 30-minute, and 1-, 2-, and 24-hour durations are plotted against recurrence interval in the top half of figures $2 \mathrm{a}-2 \mathrm{~g}$. Data points representing the three or four largest storms in the historical record are identified by year on the figures. The updated precipitation frequency curve and associated 95 percent confidence interval are superimposed over the data points so outliers may be identified. The horizontal axis on these figures is distorted such that data that is described by a Gumbel distribution will plot as a straight line. The same data, logarithmically transformed, is plotted in figures $3 a-3 g$.

Two computer-fitted lines with associated 95 percent confidence intervals--the previously defined and updated frequency curves--are presented in the bottom halves of figures $2 a-2 g$ and $3 a-3 g$. The curves show how the estimate of the magnitude of a storm for a given recurrence interval would be modified by inclusion of the August 1, 1985, storm.

Two different straight lines can be fitted through the annual maximum precipitation data points plotted on figures $2 d-2 f$ and $3 e-3 f$. One 1 ine fits the upper few data points, representing the more extreme events in the record; the other 1 ine describes the remaining data points. The calculated lines shown on each figure lie between these two lines. Two types of storms occur in Wyoming, thunderstorms and general storms; however, no distinction was made between the two types in creating figures 2 and 3 . The data were re-evaluated by identifying those storms that occurred during the major thunderstorm months of July and August of each year. The two possible fitted lines are quite distinct on figure $3 \mathrm{e}$; therefore, these data were chosen as a test case. The data were divided into three groups: (1) Storms occurring during May and June; (2) storms occurring during July and August; and (3) storms occurring during September. (None of the 1-hour annual maximum precipitation occurred during the period October through April.) Each of the data groups was identified by a different symbol and was plotted again on a copy of figure $3 e$. Except for the observation that the two largest storms were thunderstorms that occurred during the months of July and August, no consistent relationship between occurrence and month could be determined. Records were researched to determine whether the years during which the largest storms occurred coincided with possible changes in either the location, or construction, or both, of the precipitation gage. No pattern with time could be established. 

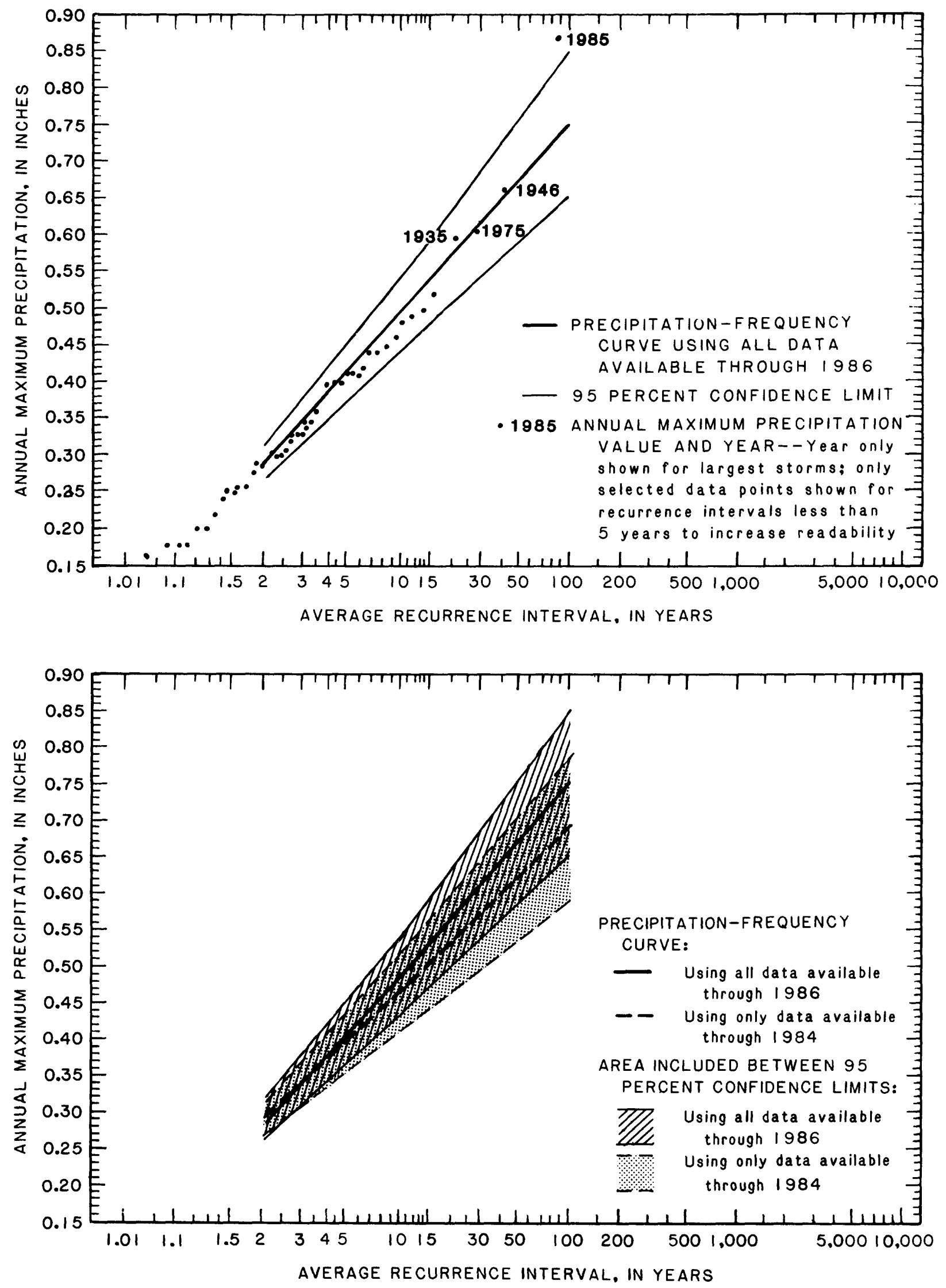

Figure 2a.--Precipitation-frequency curves for 5-minute duration, arithmetic Gumbel distribution. 


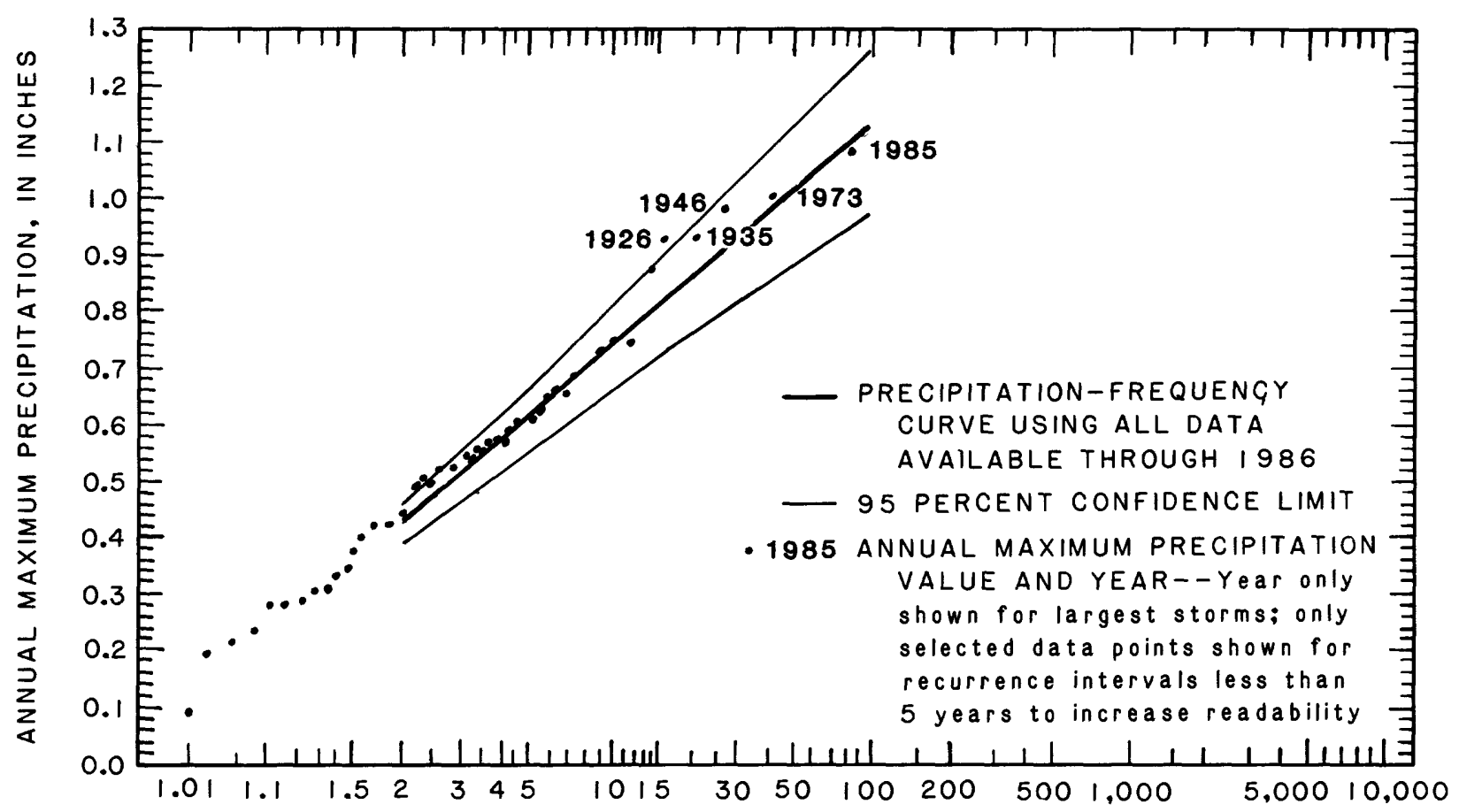

AVERAGE RECURRENCE INTERVAL, IN YEARS

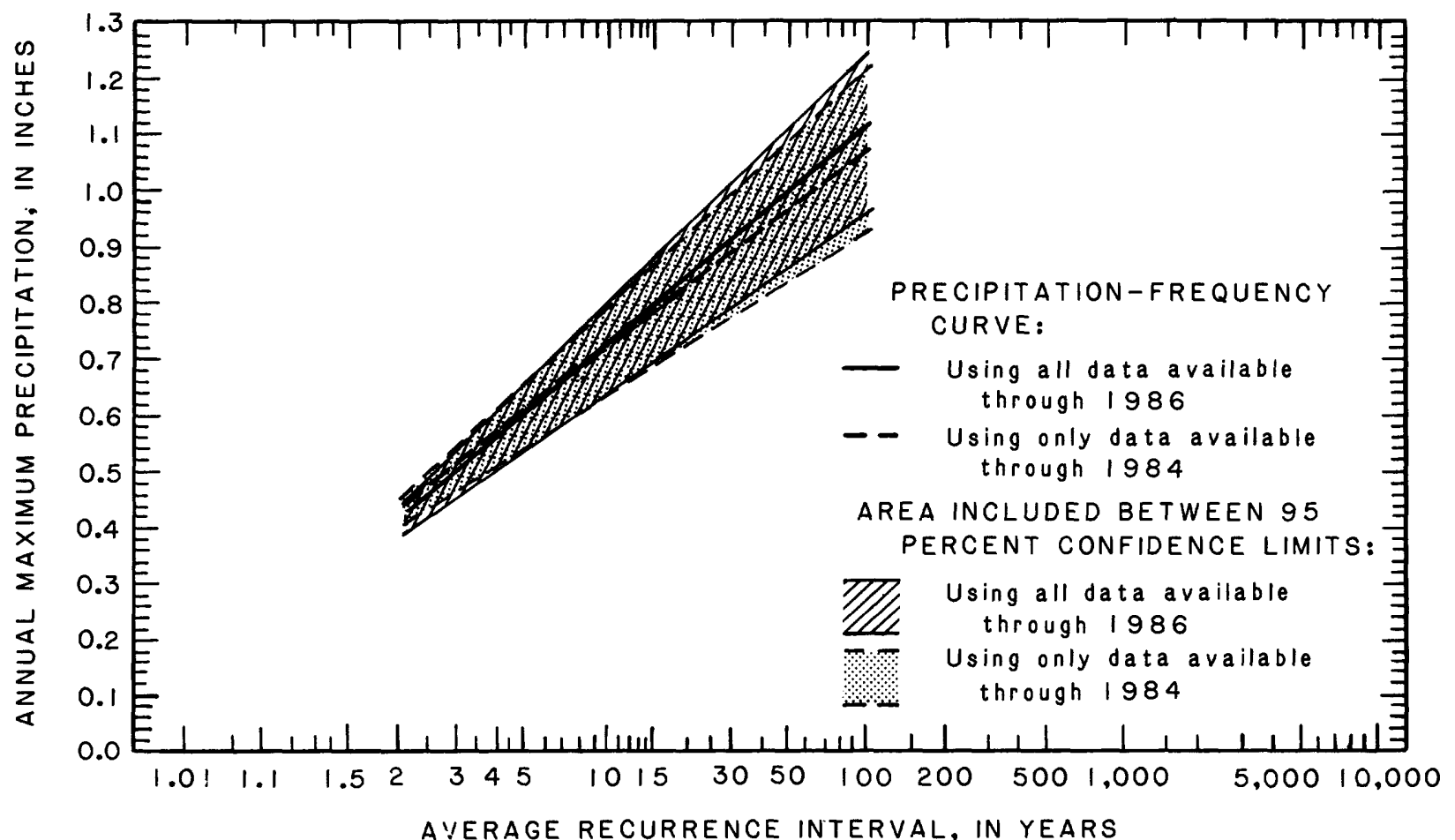

Figure 2b.--Precipitation-frequency curves for 10-minute duration, arithmetic Gumbel distribution. 

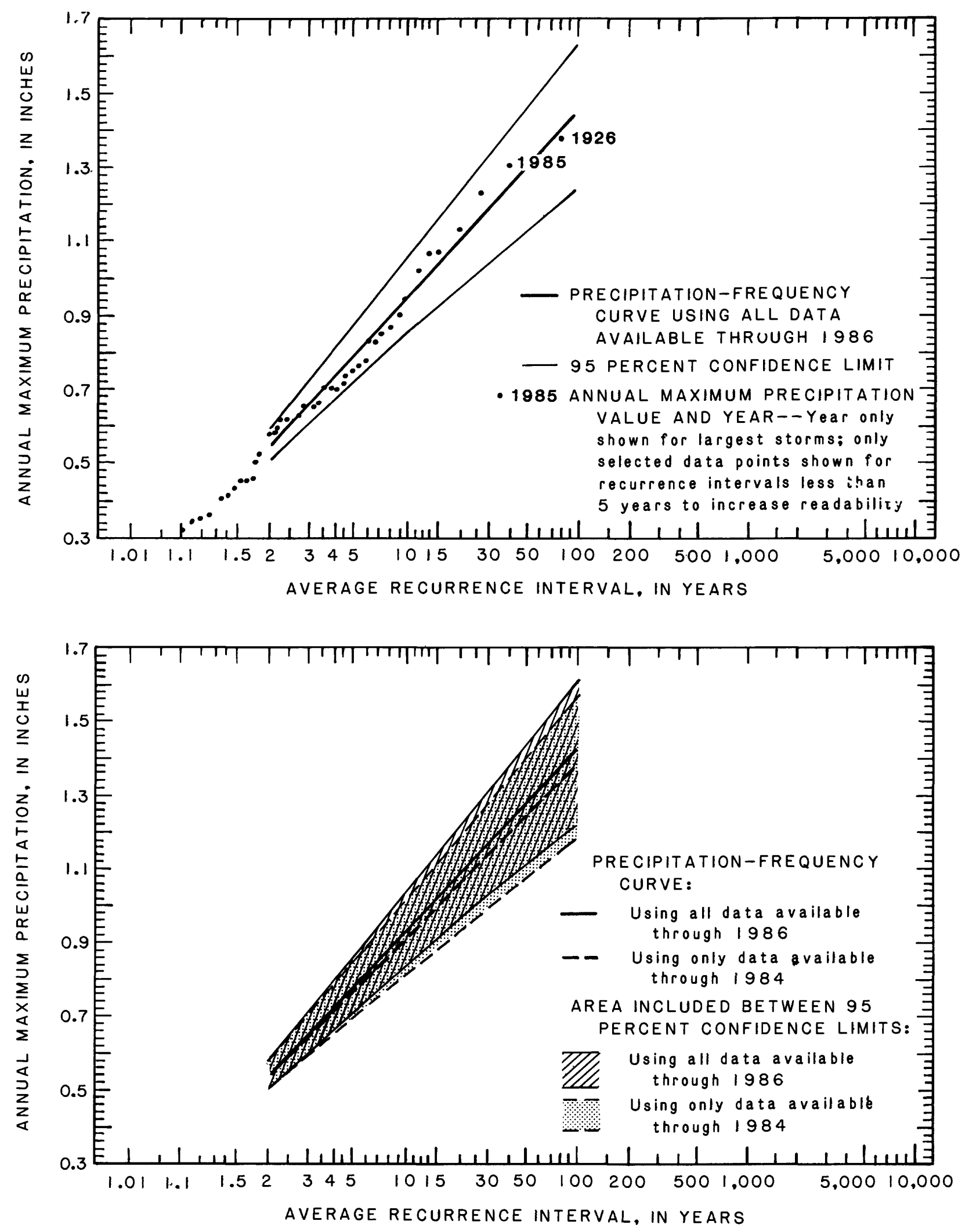

Figure 2c.--Precipitation-frequency curves for 15-minute duration, arithmetic Gumbel distribution. 


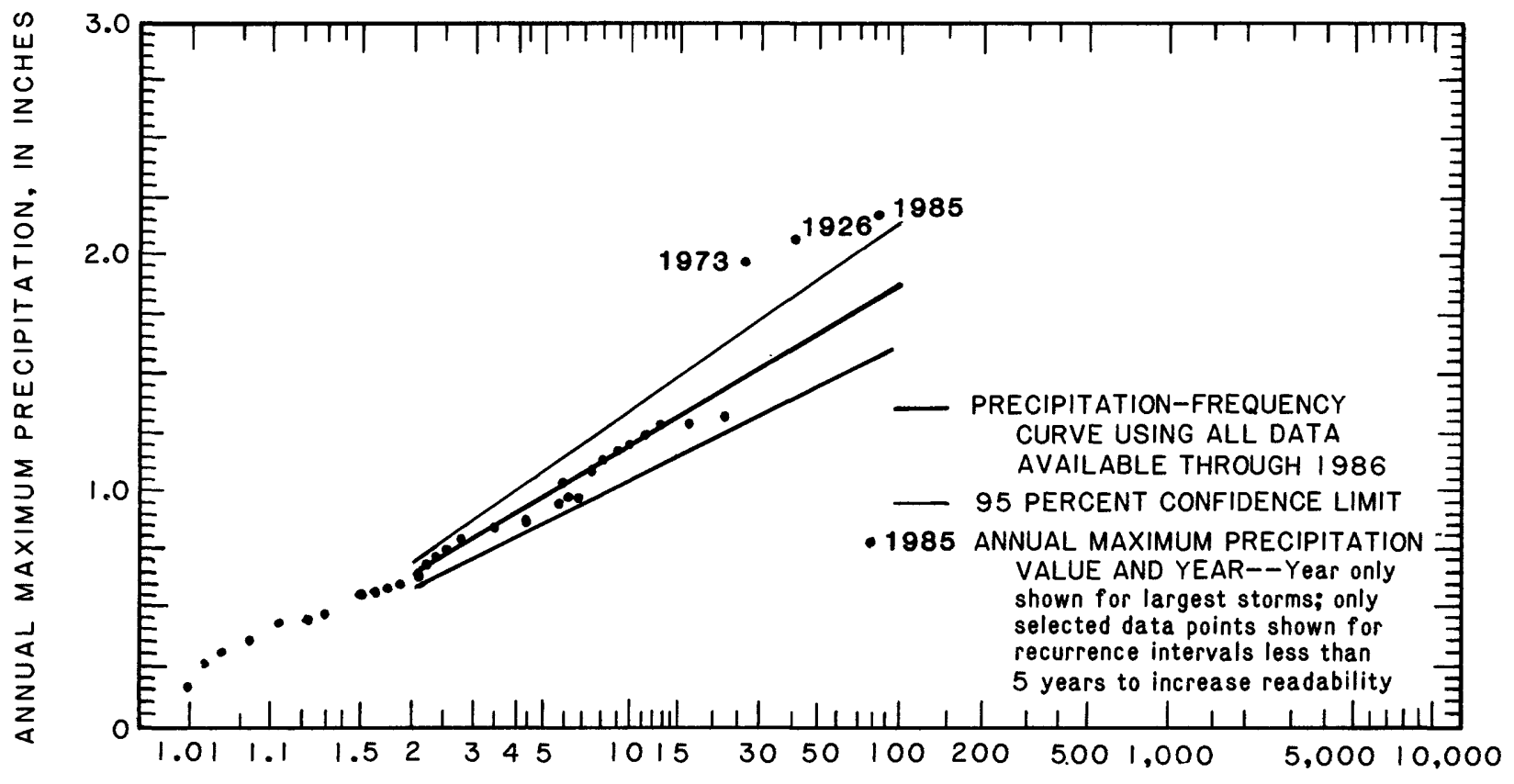

AVERAGE RECURRENCE INTERVAL, IN YEARS

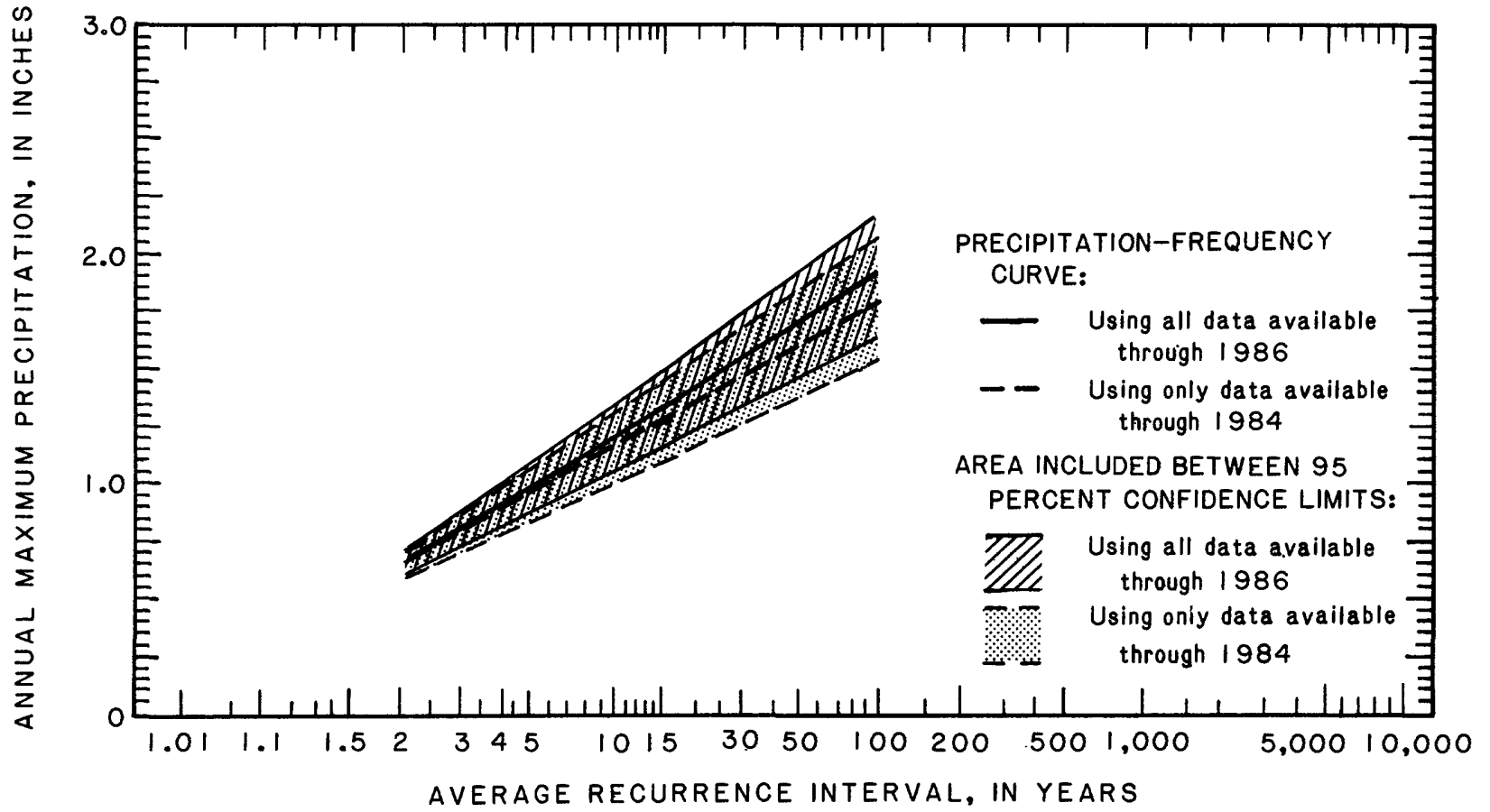

Figure 2d.--Precipitation-frequency curves for 30-minute duration, arithmetic Gumbel distribution. 

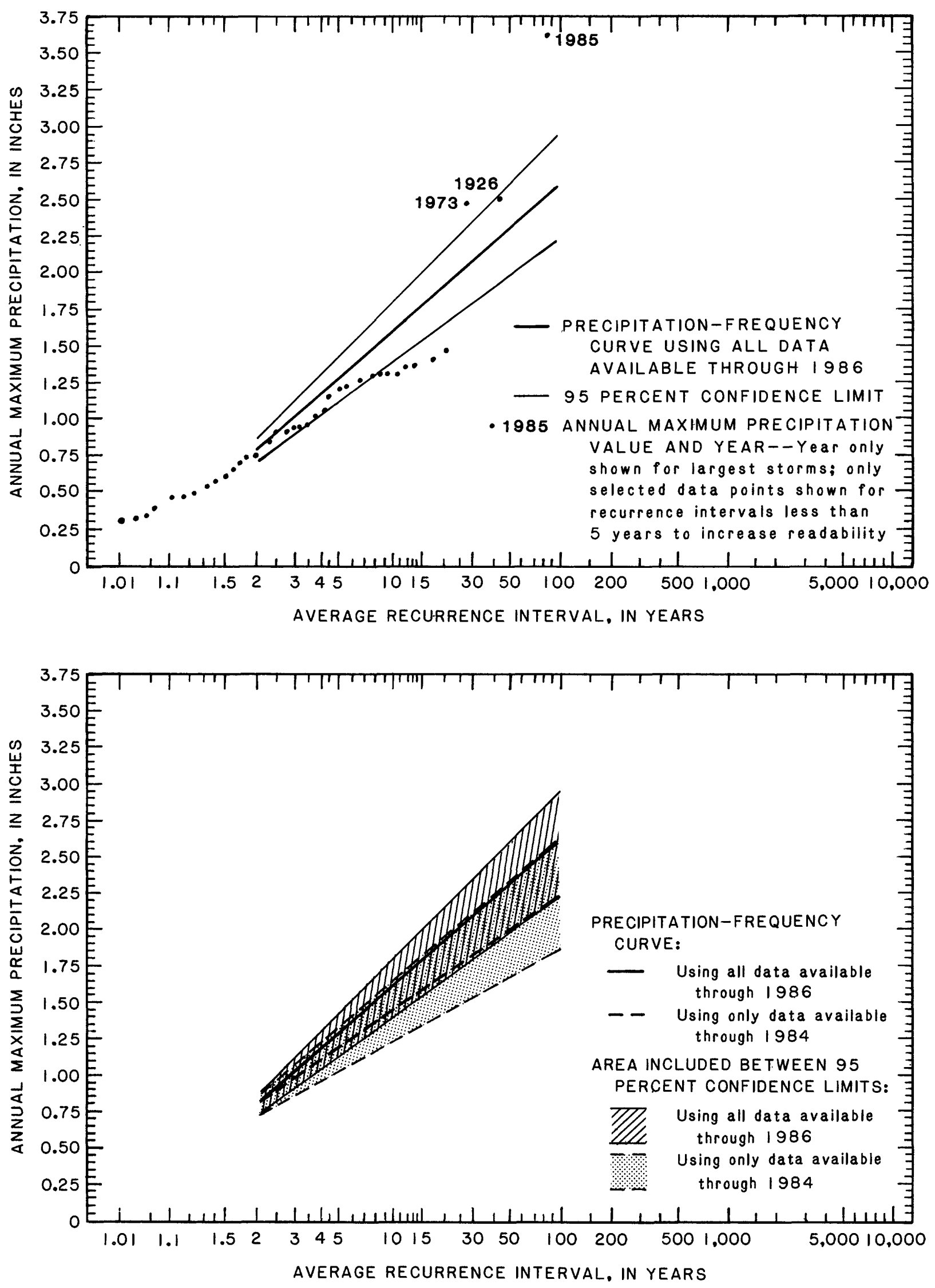

Figure 2e.--Precipitation-frequency curves for 1-hour duration, arithmetic Gumbel distribution. 


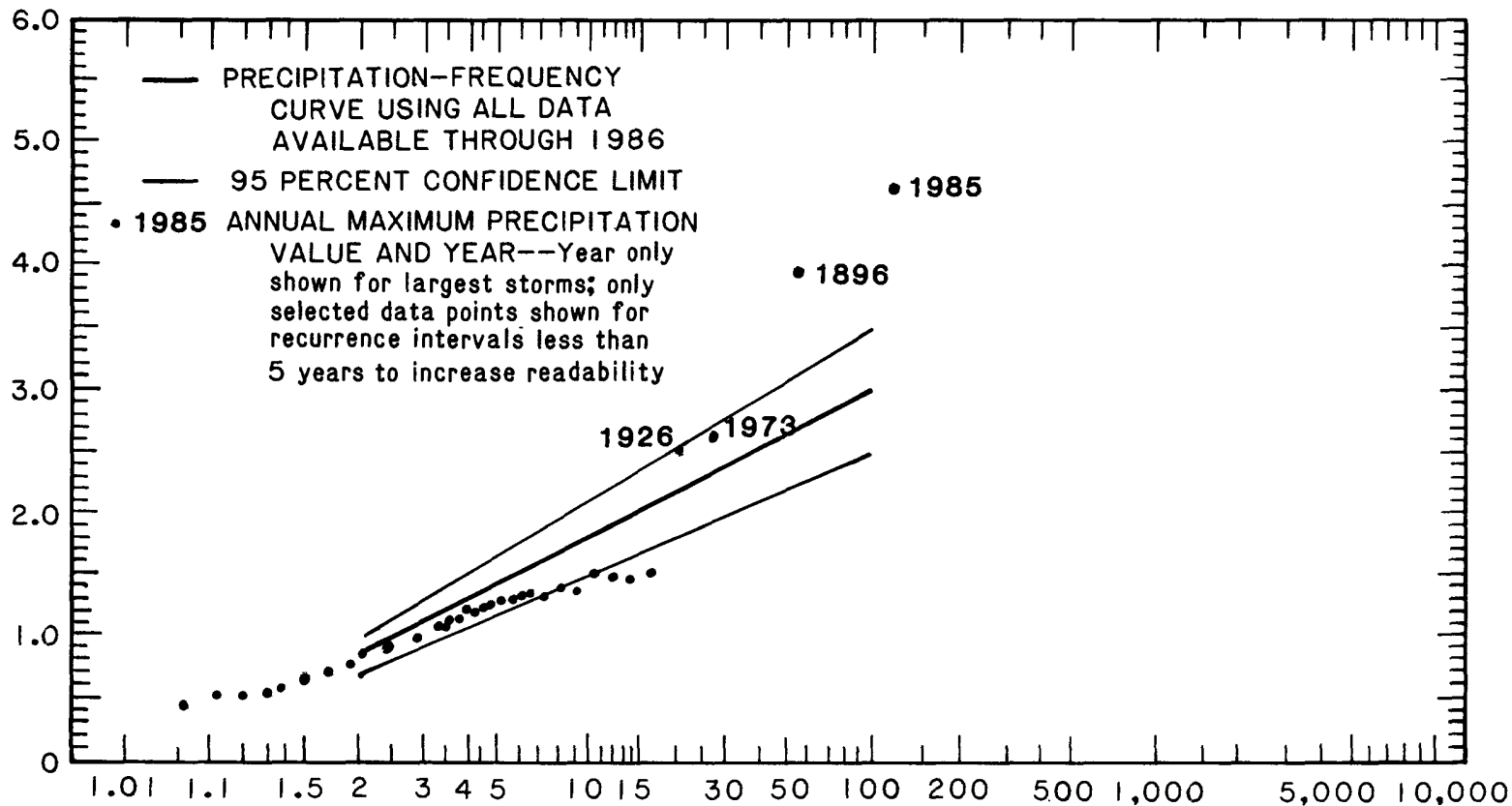

AVERAGE RECURRENCE INTERVAL, IN YEARS

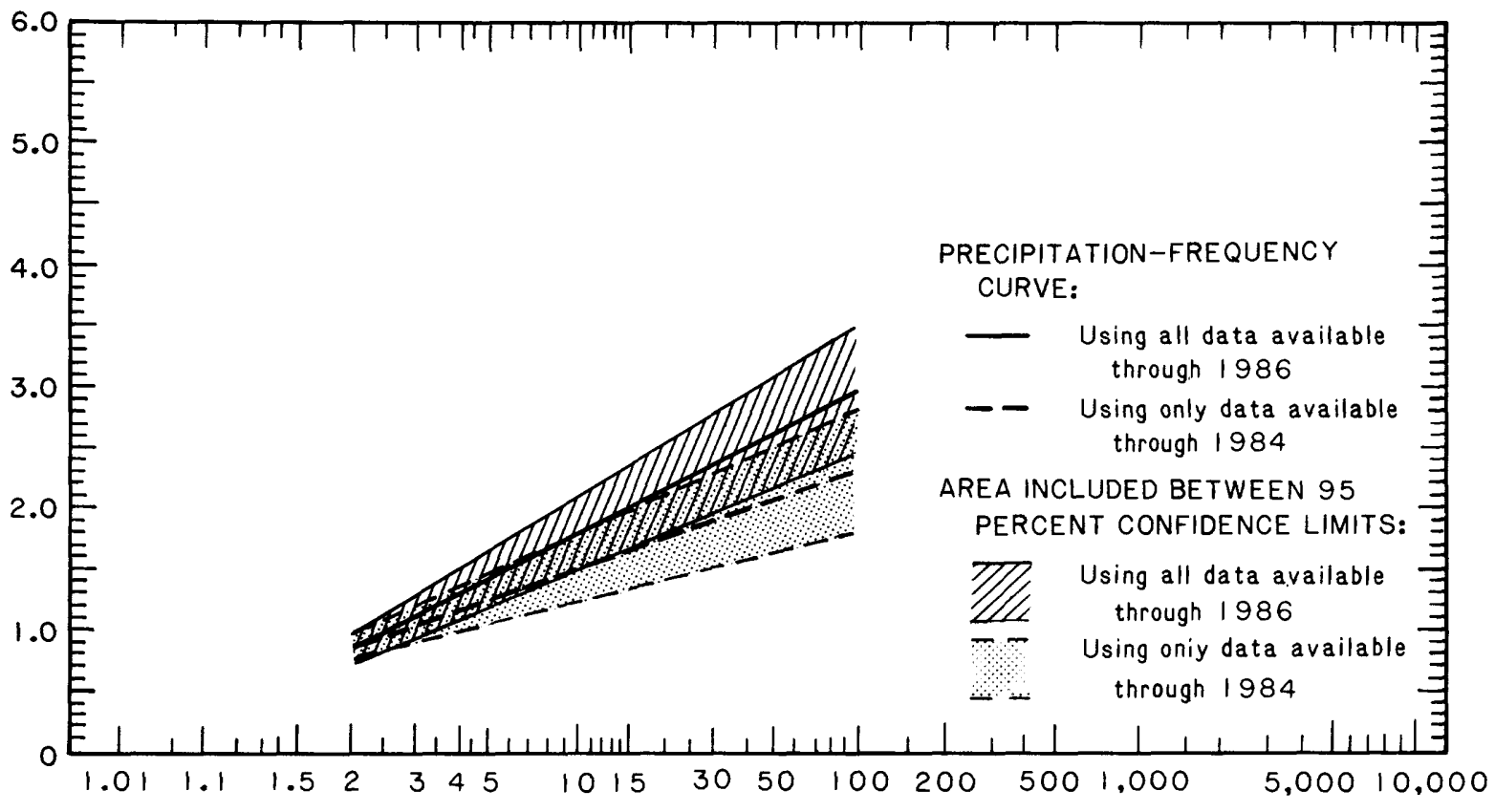

AVERAGE RECURRENCE INTERVAL, IN YEARS

Figure 2 f.--Precipitation-frequency curves for 2-hour duration, arithmetic Gumbel distribution. 

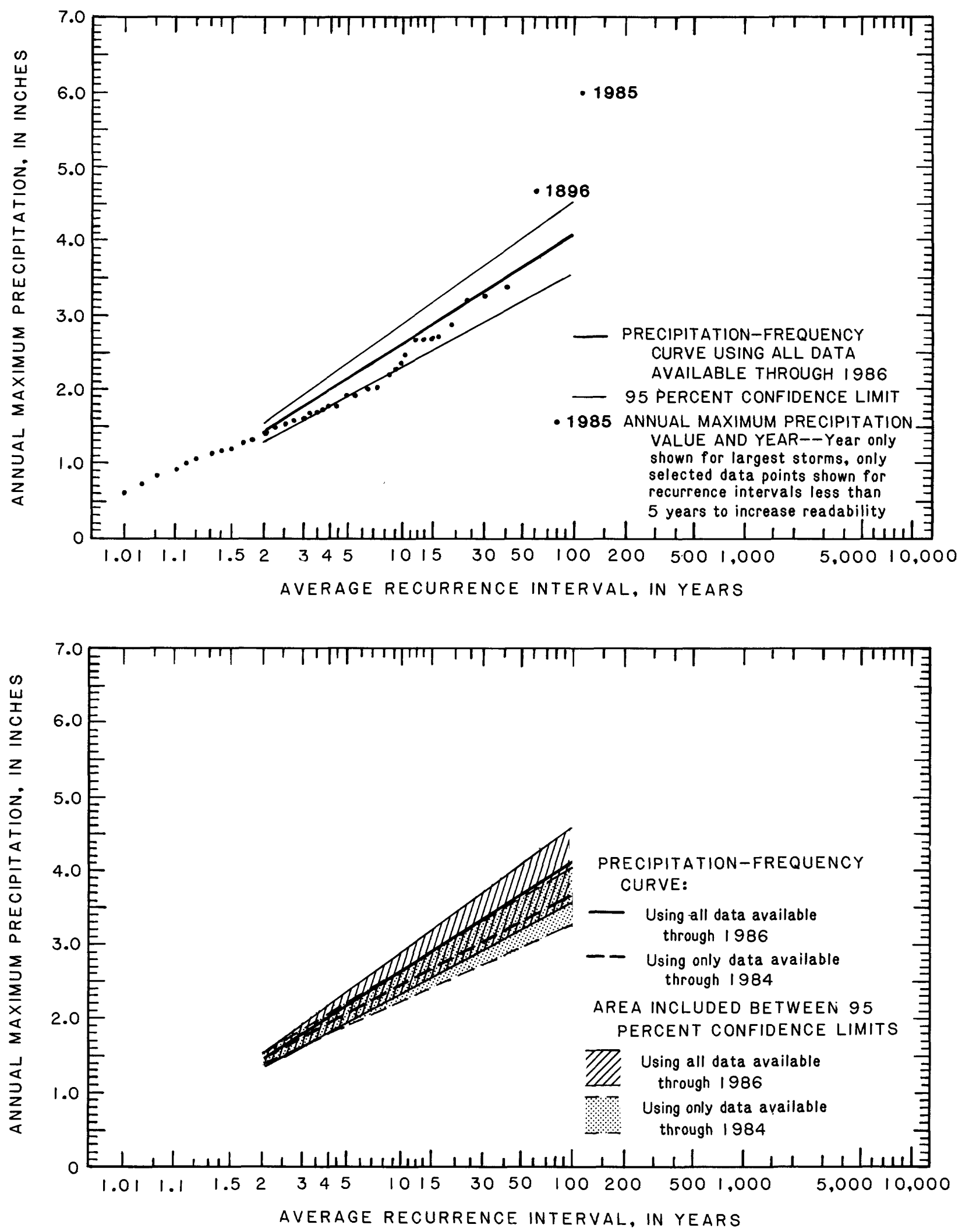

Figure 2g.--Precipitation-frequency curves for 24-hour duration, arithmetic Gumbel distribution. 


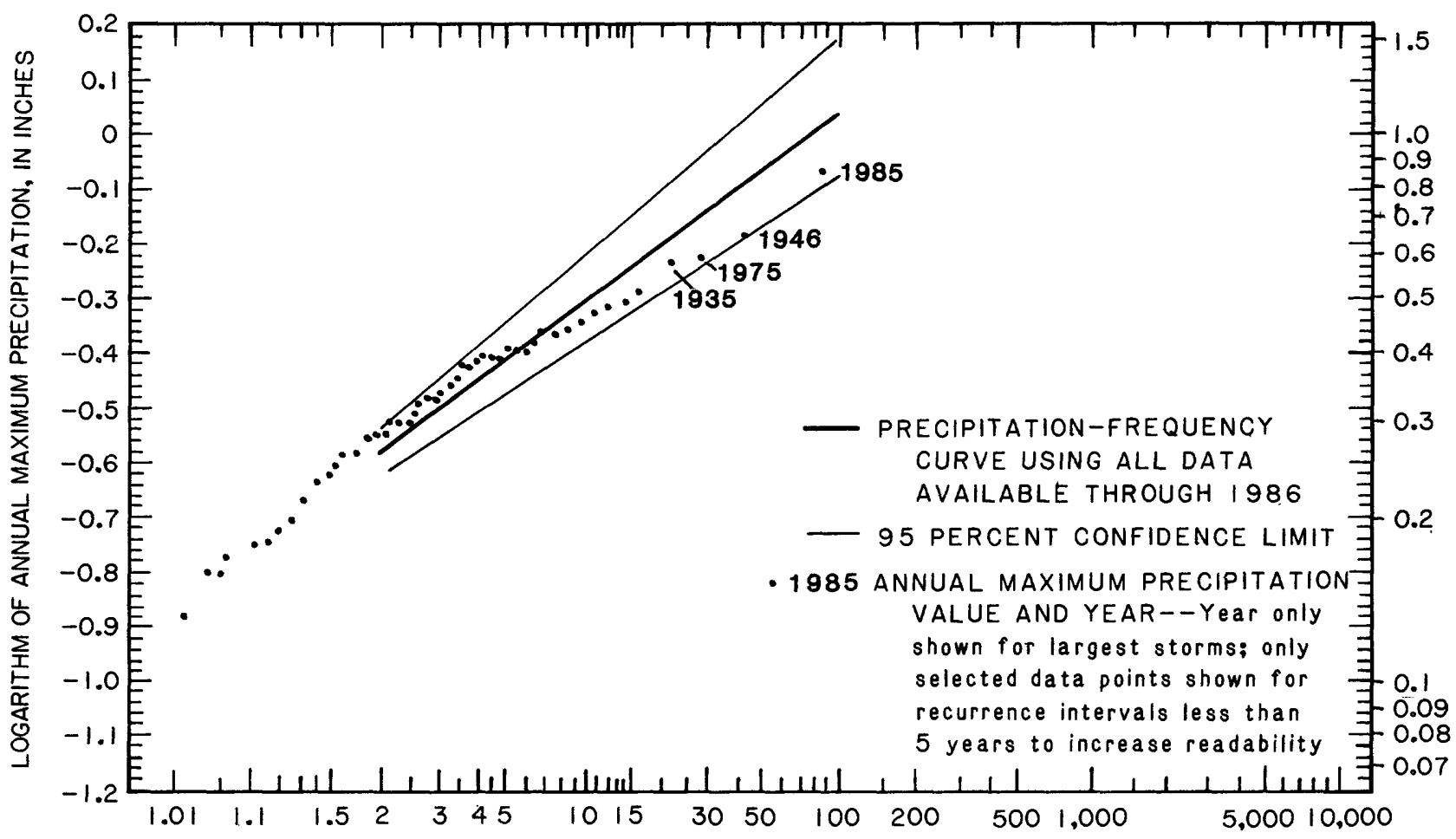

AVERAGE RECURRENCE INTERVAL, IN YEARS

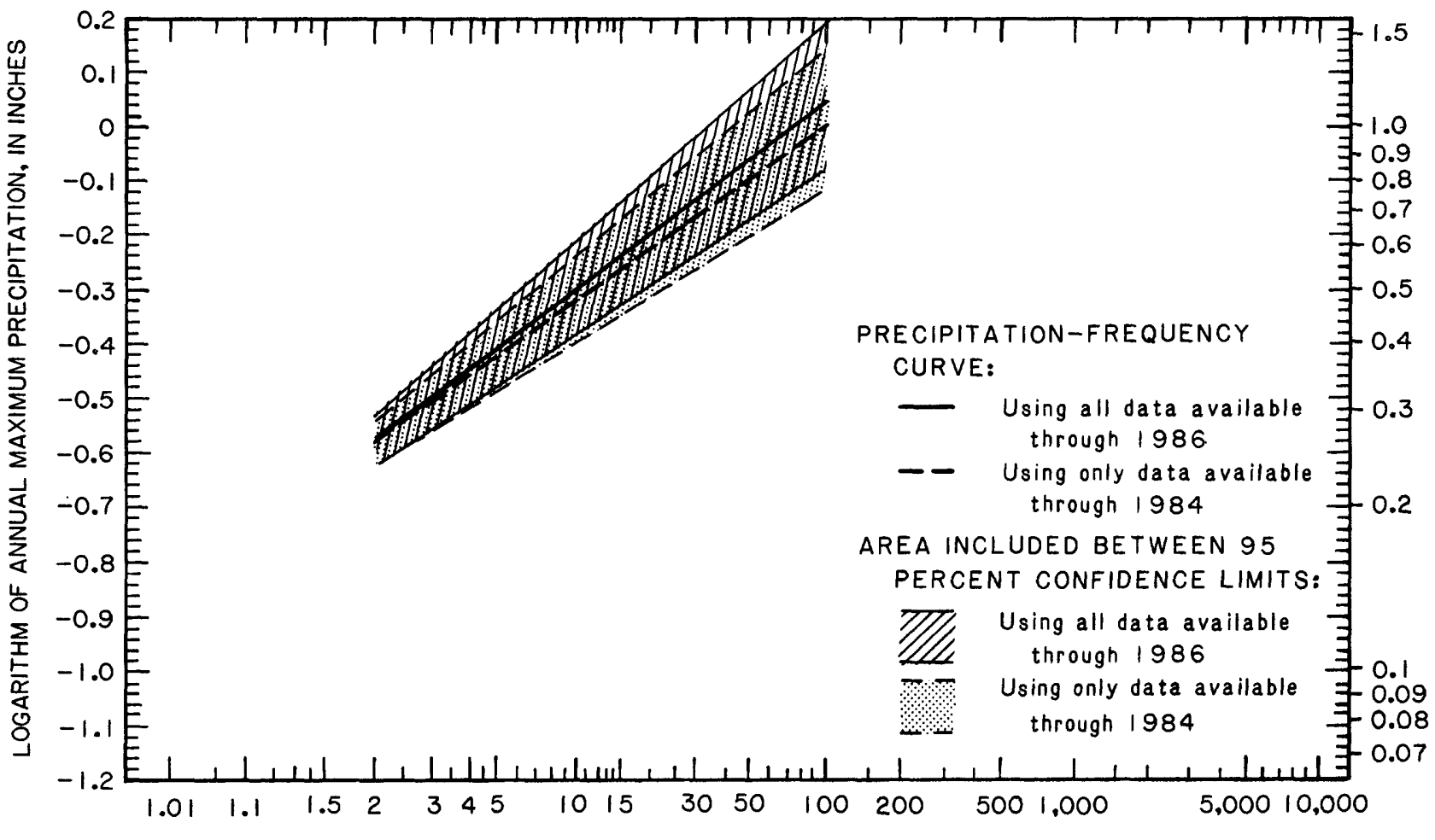

Figure 3a.--Precipitation-frequency curves for 5-minute duration, logarithmic Gumbel distribution. 

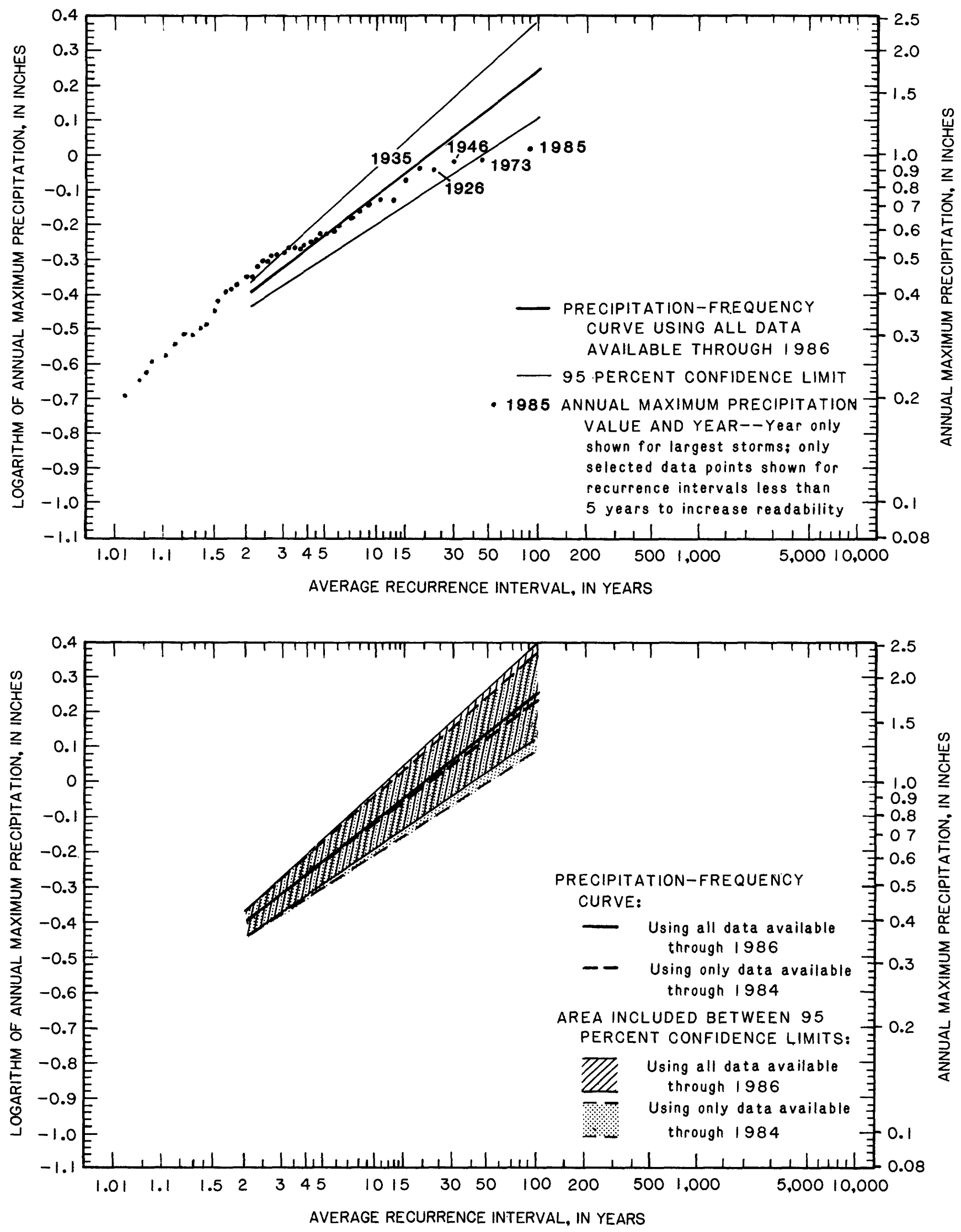

Figure 3b.--Precipitation-frequency curves for 10-minute duration, logarithmic Gumbel distribution. 

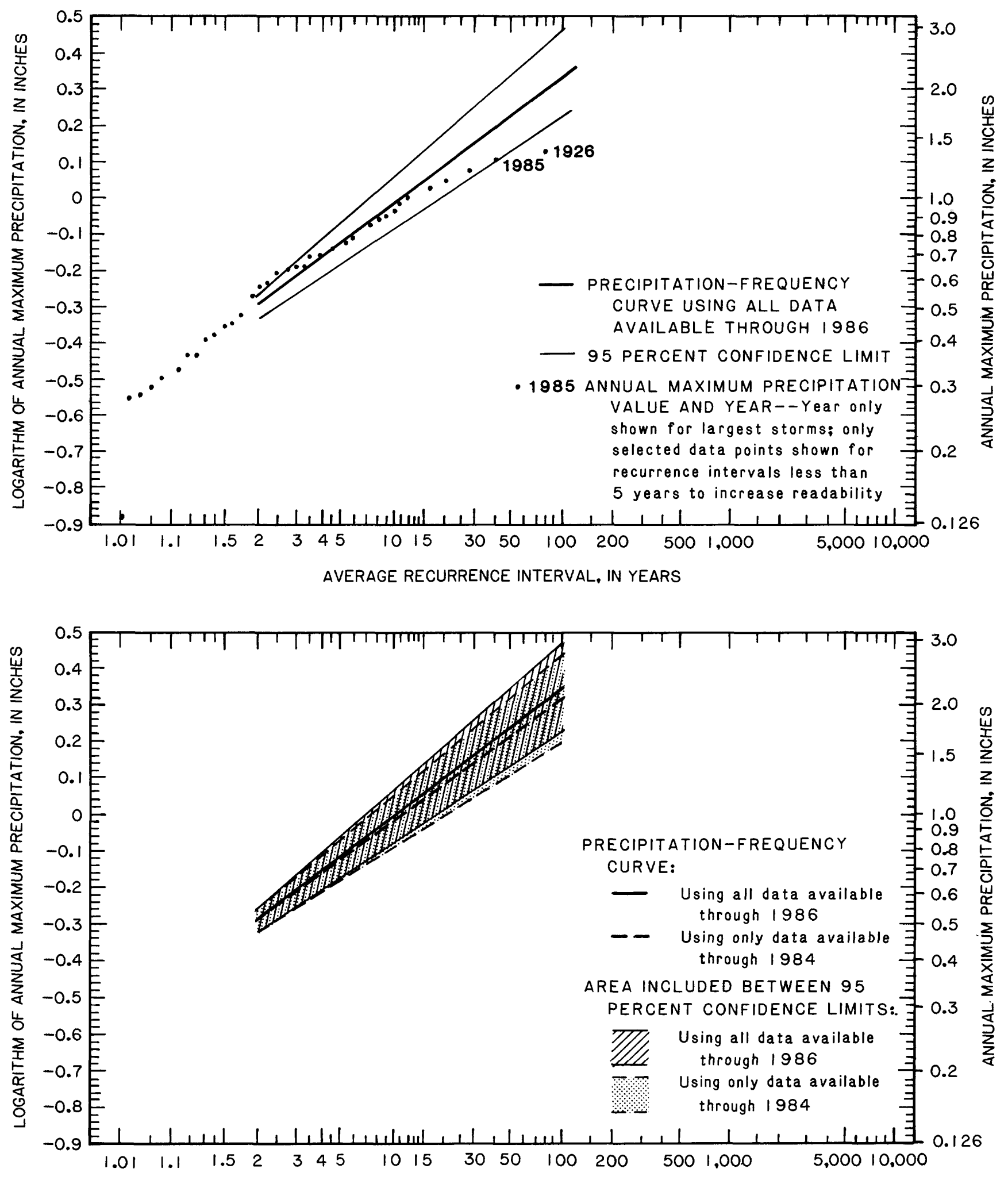

AVERAGE RECURRENCE INTERVAL, IN YEARS

Figure 3c.--Precipitation-frequency curves for 15-minute duration, logarithmic Gumbel distribution. 

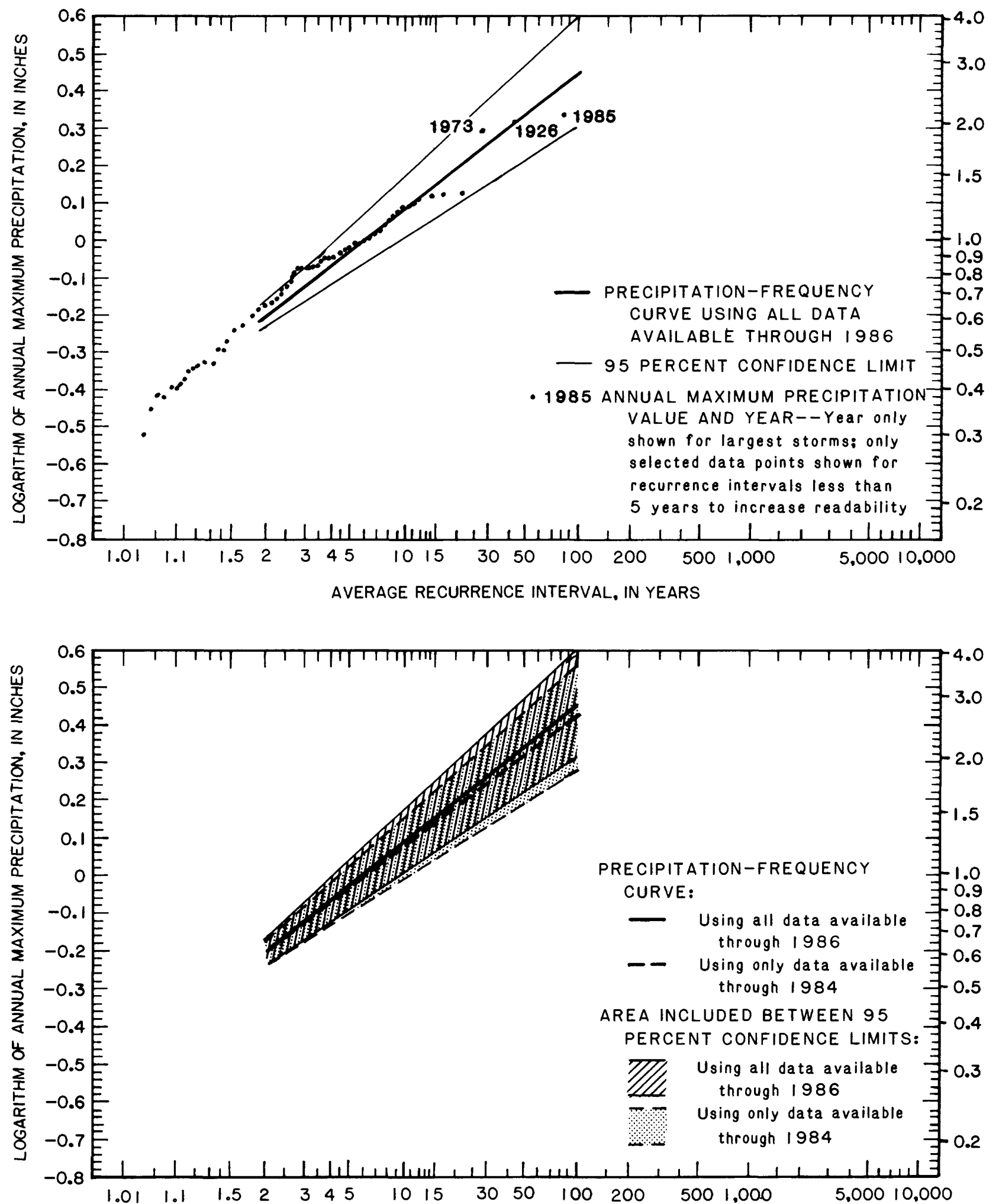

Figure 3d.--Precipitation-frequency curves for 30-minute duration, logarithmic Gumbel distribution. 

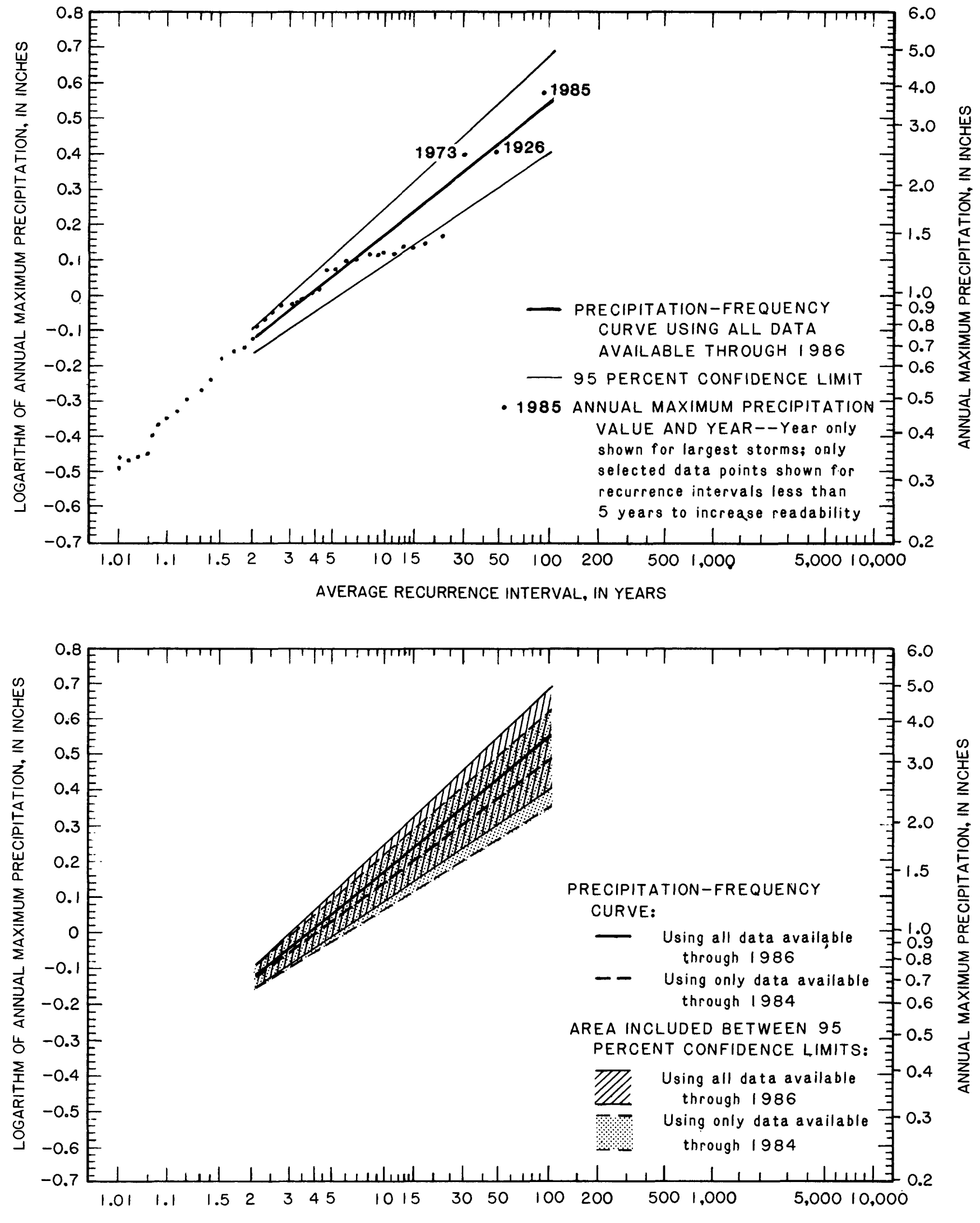

AVERAGE RECURRENCE INTERVAL, IN YEARS

Figure 3e.--Precipitation-frequency curves for 1-hour duration, logarithmic Gumbel distribution. 

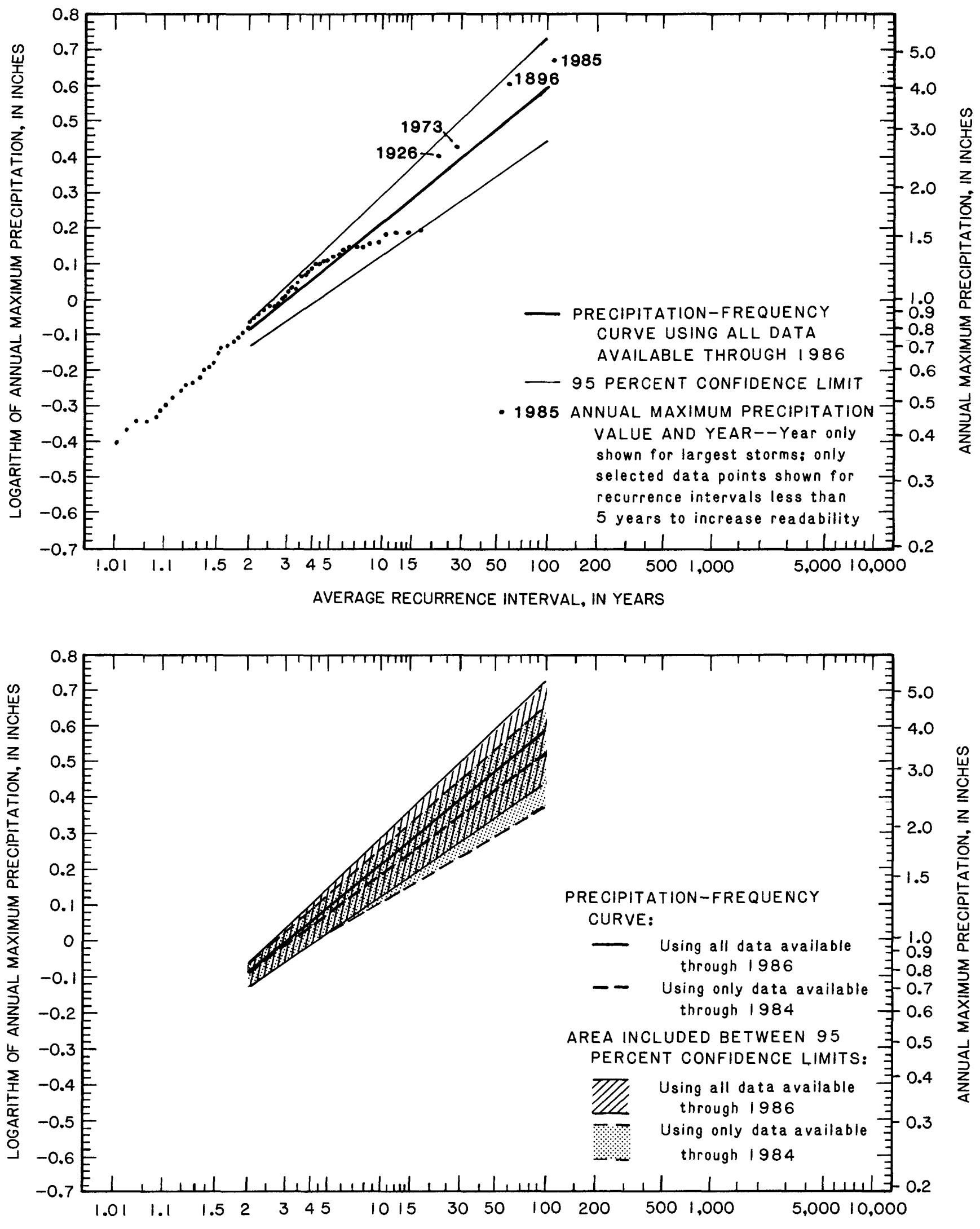

AVERAGE RECURRENCE INTERVAL, IN YEARS

Figure $3 \mathrm{f}$.--Precipitation-frequency curves for 2-hour duration, logarithmic Gumbel distribution. 


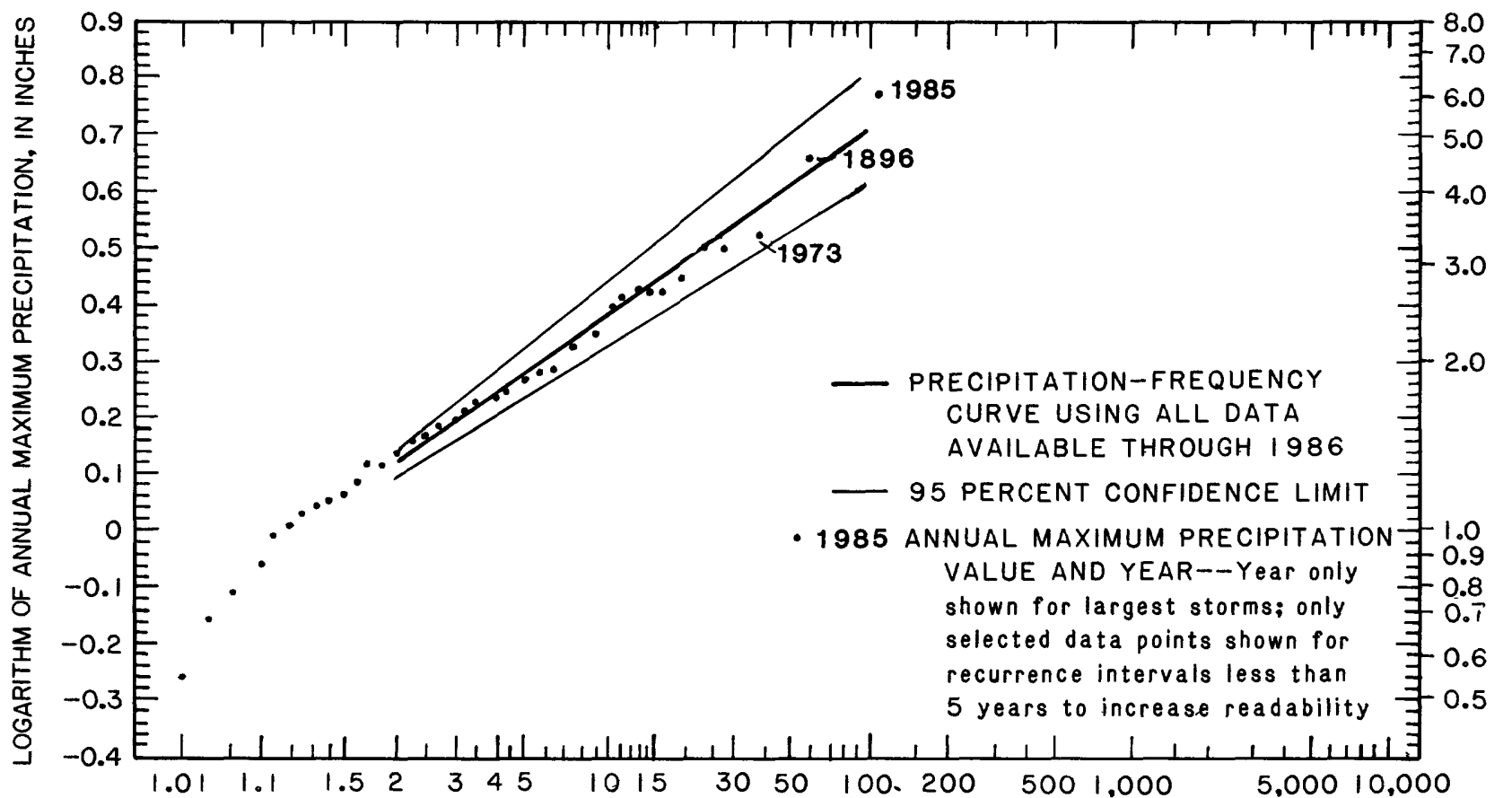

AVERAGE RECURRENCE INTERVAL, IN YEARS

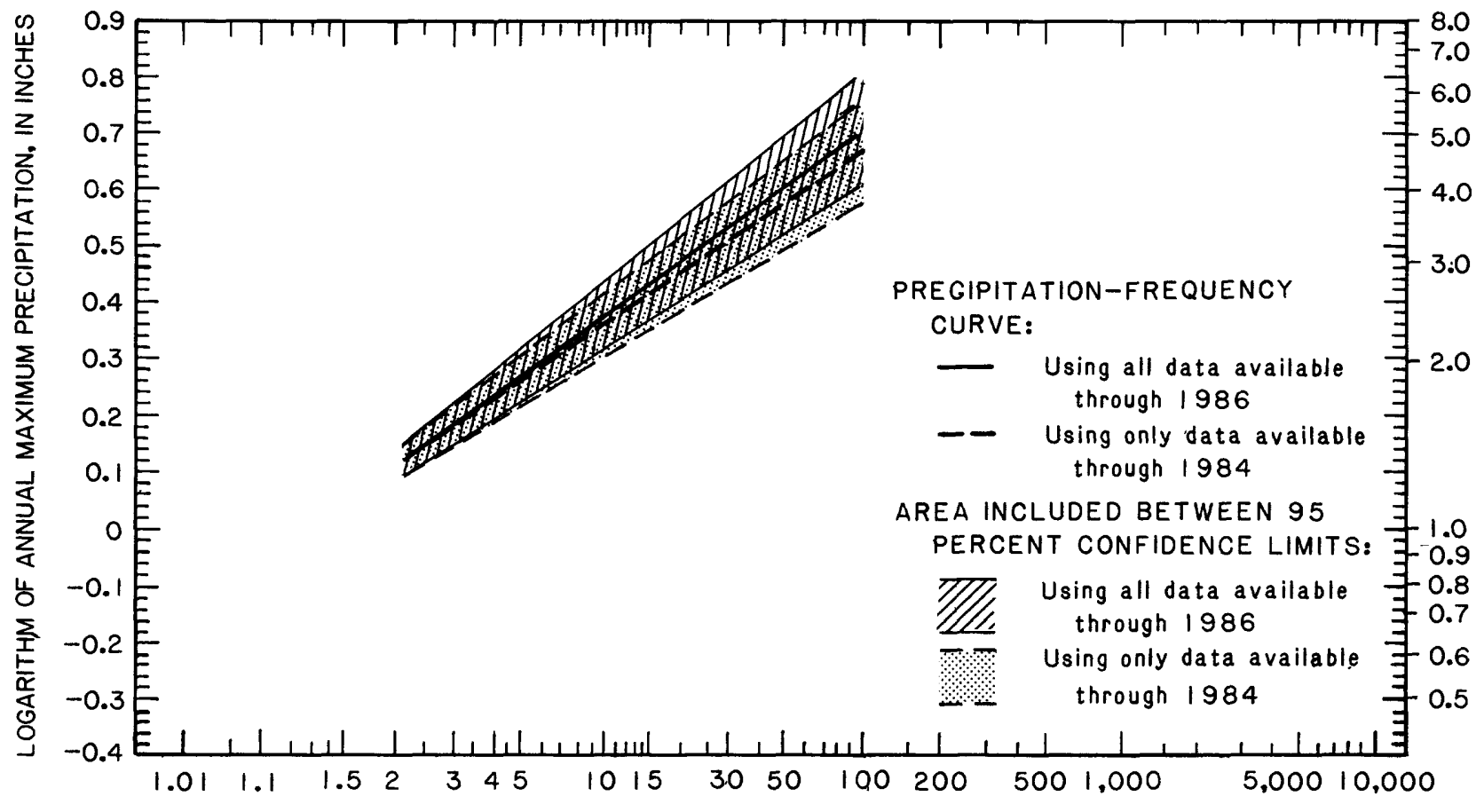

Figure 3g.--Precipitation-frequency curves for 24-hour duration, logarithmic Gumbel distribution. 
Fitted lines based on the assumption that the logarithms of the data are described by the log-Pearson Type III distribution are plotted in figure 4. It is impractical to develop a horizontal axis for this distribution that is distorted such that data would plot as a straight 1 ine, as was the case with the Gumbel distribution, because a different distortion would have to be developed for each value of skewness. Instead, data points are connected by a smooth curve.

The prediction of the magnitude of any event with a recurrence interval of 10 years or less is relatively insensitive to the distribution used--the predictions made using the three different distributions agree within 10 percent. This insensitivity is due to the fact that the period of record is long compared to the recurrence interval being considered (Frank Richards, National Weather Service, oral commun., 1987). In contrast, the predicted magnitude of events with recurrence intervals of 100 years or longer is quite sensitive to the distribution used. For example, the predicted magnitude of the 5-minute, 100-year event is more than 50 percent larger if the $10 \mathrm{~g}$-Gumbel distribution is used to describe the data than if either the arithmetic Gumbel or the log-Pearson Type III distribution is used.

For durations where the August 1, 1985, storm was an outlier ( 5 minutes, 1 hour, 2 hours, and 24 hours), the predicted magnitudes of events with long recurrence intervals are sensitive to whether or not data from 1985 and 1986 were used in the analysis. This sensitivity is an indication of how much weight the various distributions give to the outlying point that represents the 1985 storm. Because the 1985 storm was not an outlier for the 10- and 15-minute durations, the calculated magnitudes for those durations is almost the same regardless of whether the previously defined or updated distribution is used, even for events with very long recurrence intervals.

\section{INTENSITY-DURATION CURVES}

Intensity-duration curves for storms of selected recurrence intervals were developed using regional equations, and the previously defined and updated Gumbe1, log-Gumbel, and log-Pearson Type III analyses. The calculated curves were then compared to the observed intensity-duration curve for the storm of August 1, 1985.

\section{Method of Analysis}

Stee1 and McGhee (1979, p. 326-327) divide the United States into seven areas and present regionalized equations of intensity-duration curves for selected recurrence intervals. The authors advise that these equations should be used with caution since wide variations in precipitation patterns are to be expected over such large sections of the country. However, the curves can serve as useful preliminary estimates. The 100-year-storm intensity-duration curve calculated from the Steel and McGhee equations for area 5 (that includes southeastern Wyoming) is plotted on figure 5. 

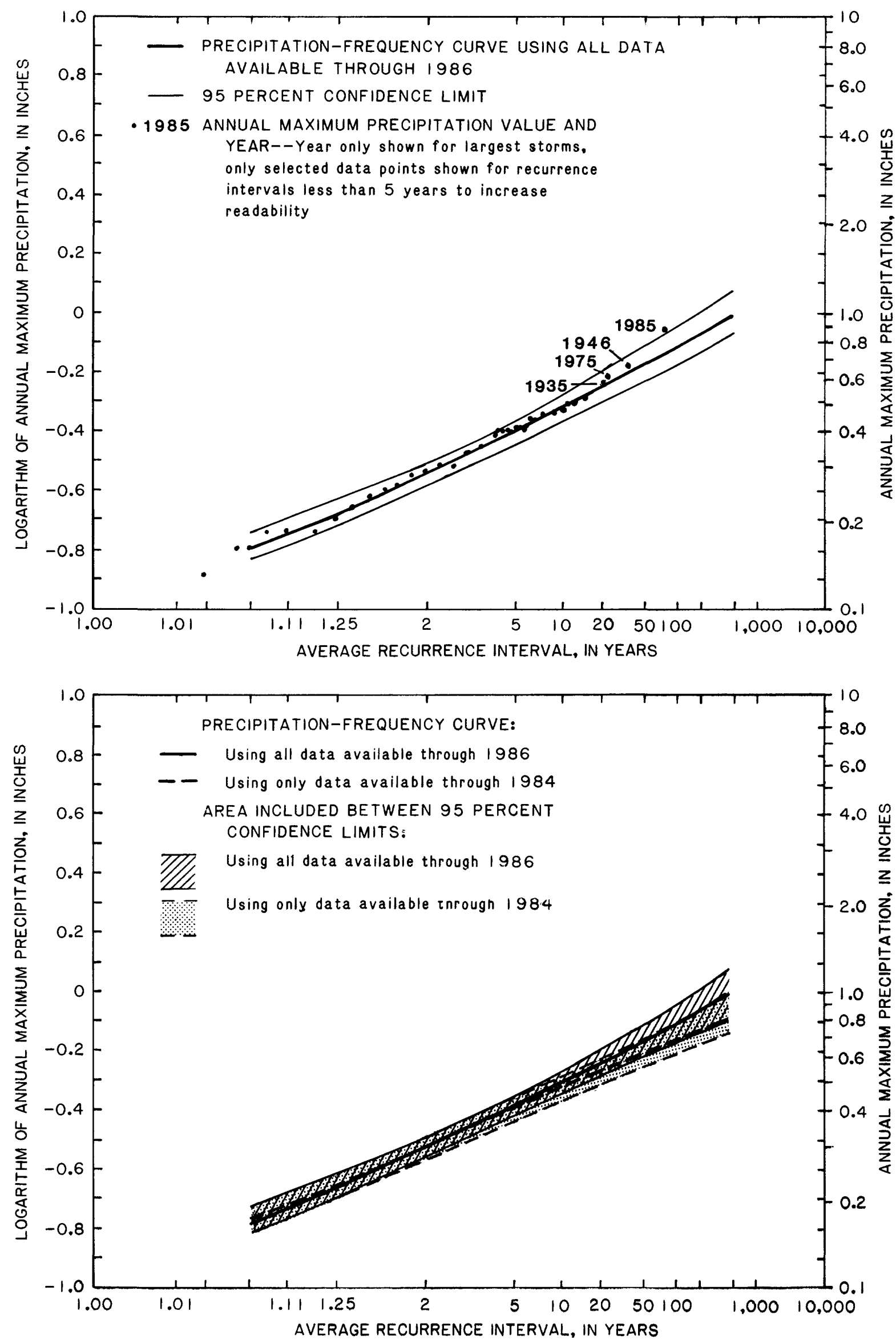

Figure 4a.--Precipitation-frequency curves for 5-minute duration, Pearson Type III distribution. 

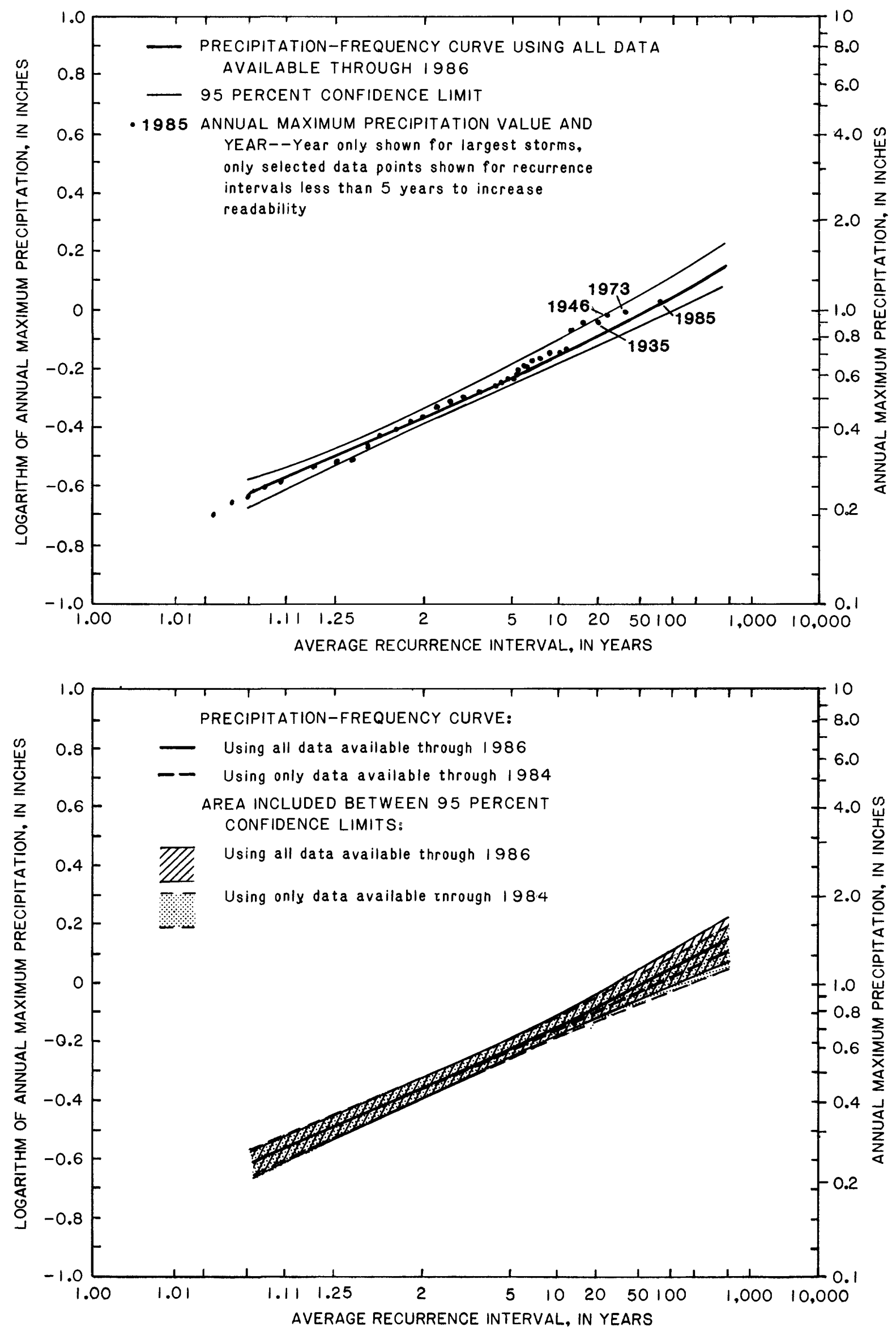

Figure 4b.--Precipitation-frequency curves for 10-minute duration, Pearson Type III distribution. 

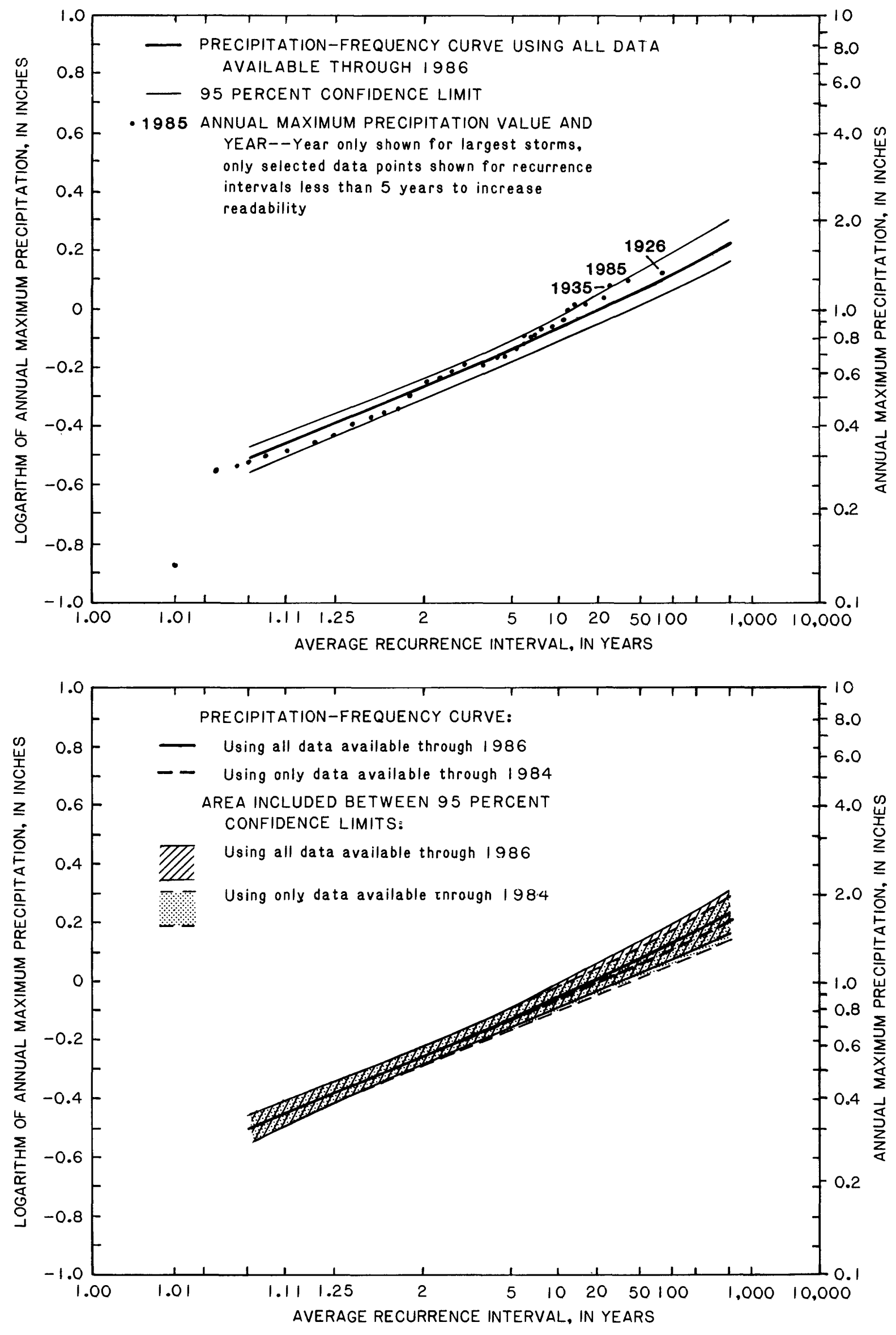

Figure 4c.--Precipitation-frequency curves for 15-minute duration, Pearson Type III distribution. 

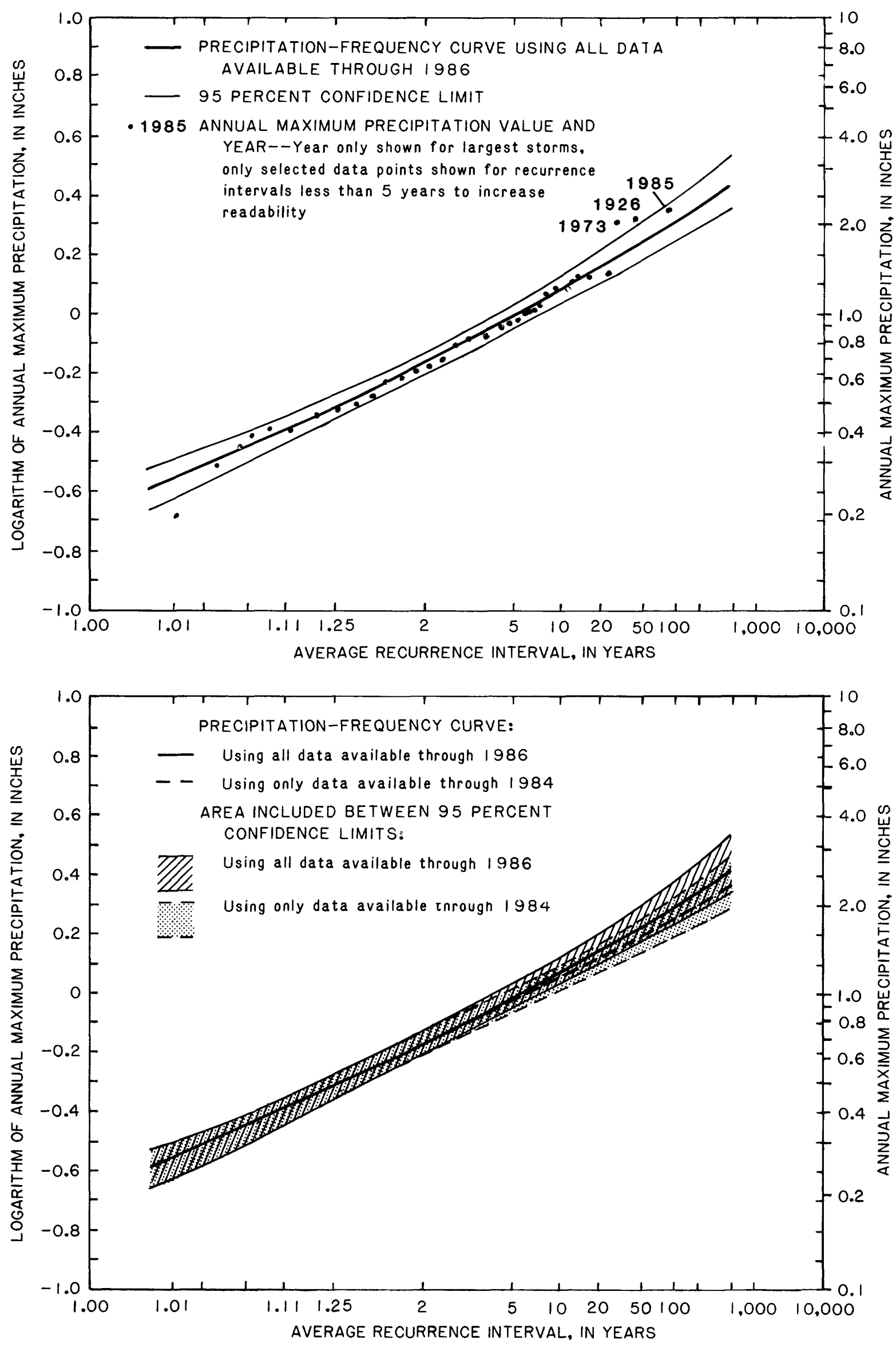

Figure 4d.--Precipitation-frequency curves for 30-minute duration, Pearson Type III distribution. 

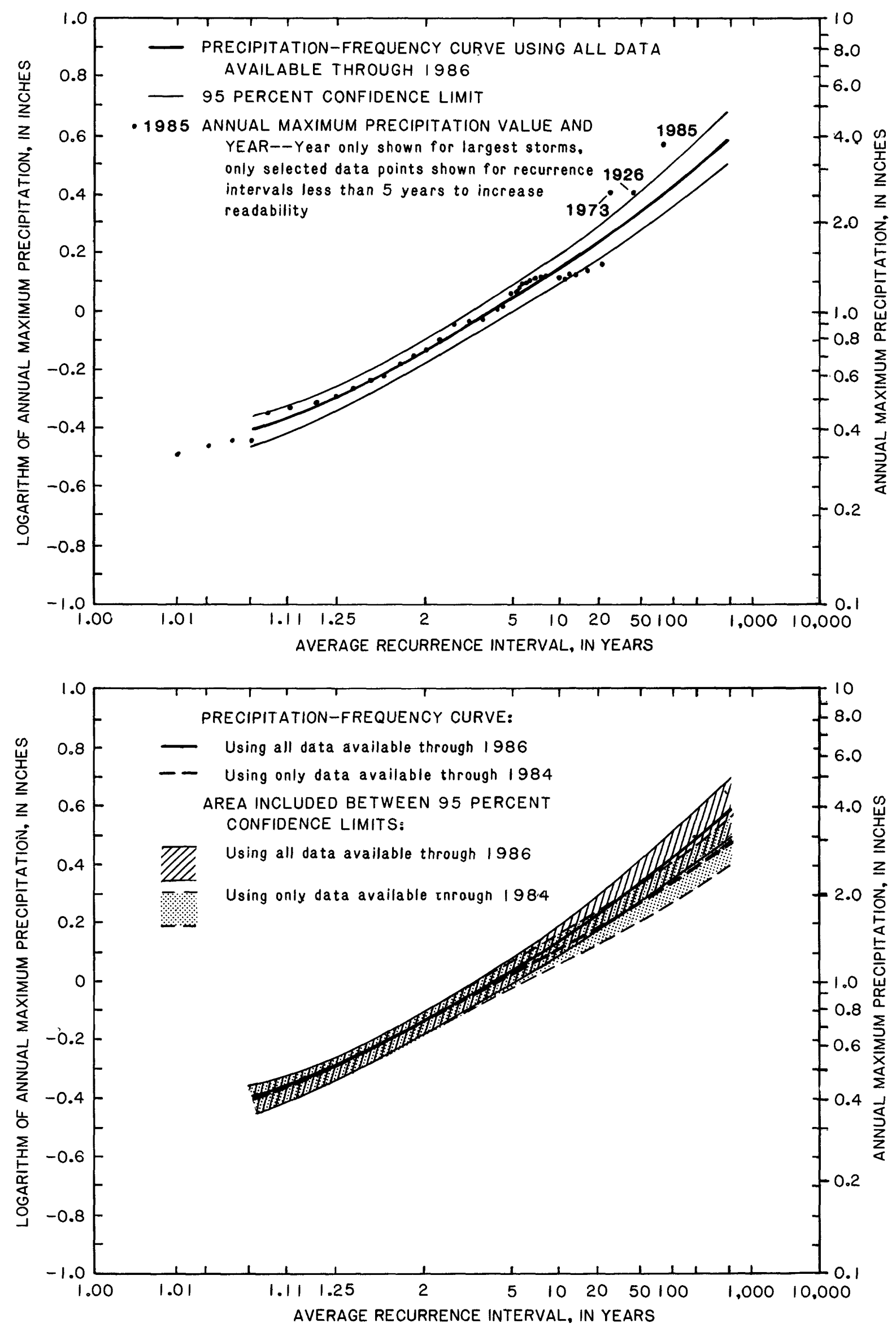

Figure 4e.--Precipitation-frequency curves for 1-hour duration, Pearson Type III distribution. 

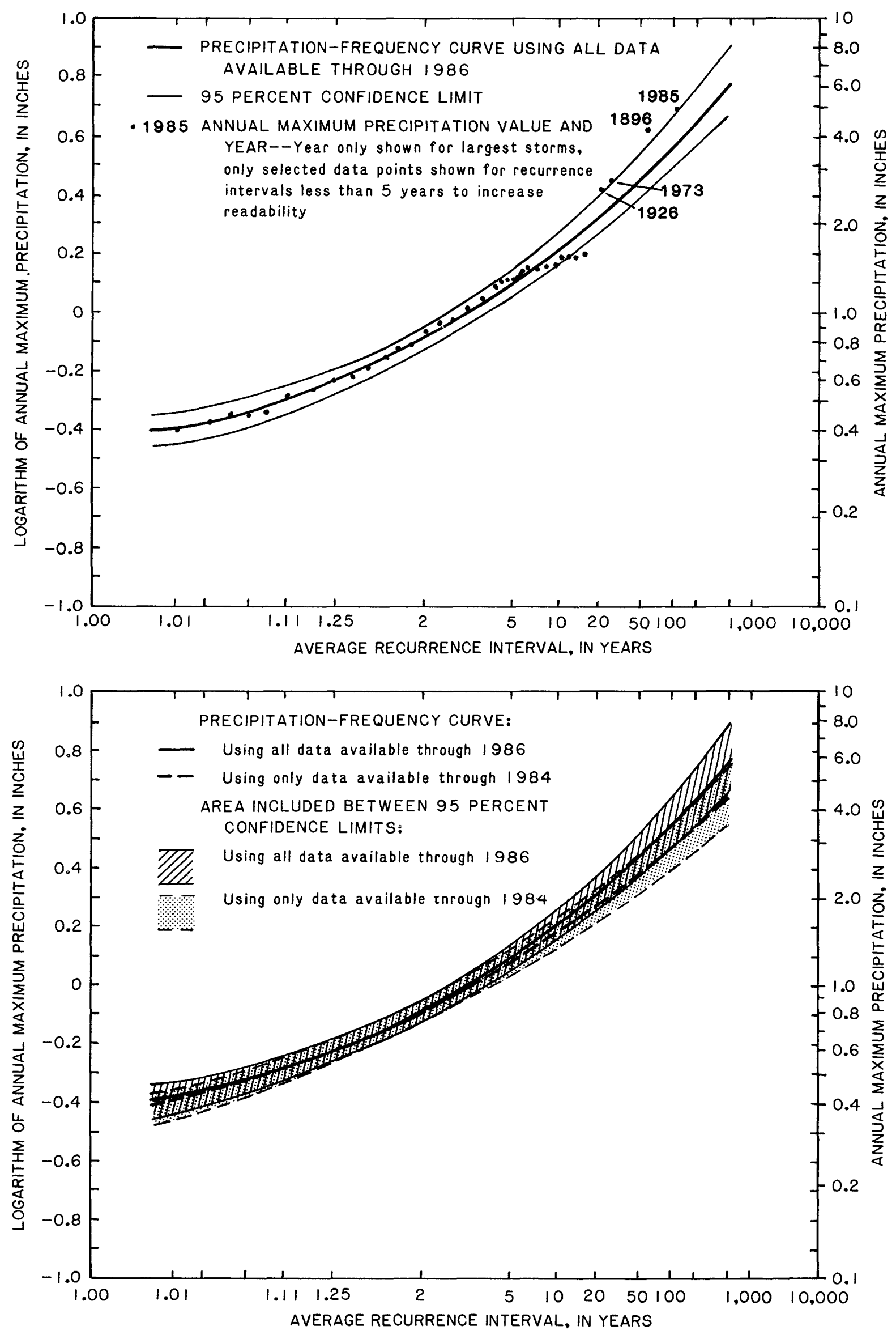

Figure 4 f.--Precipitation-frequency curves for 2-hour duration, Pearson Type III distribution. 

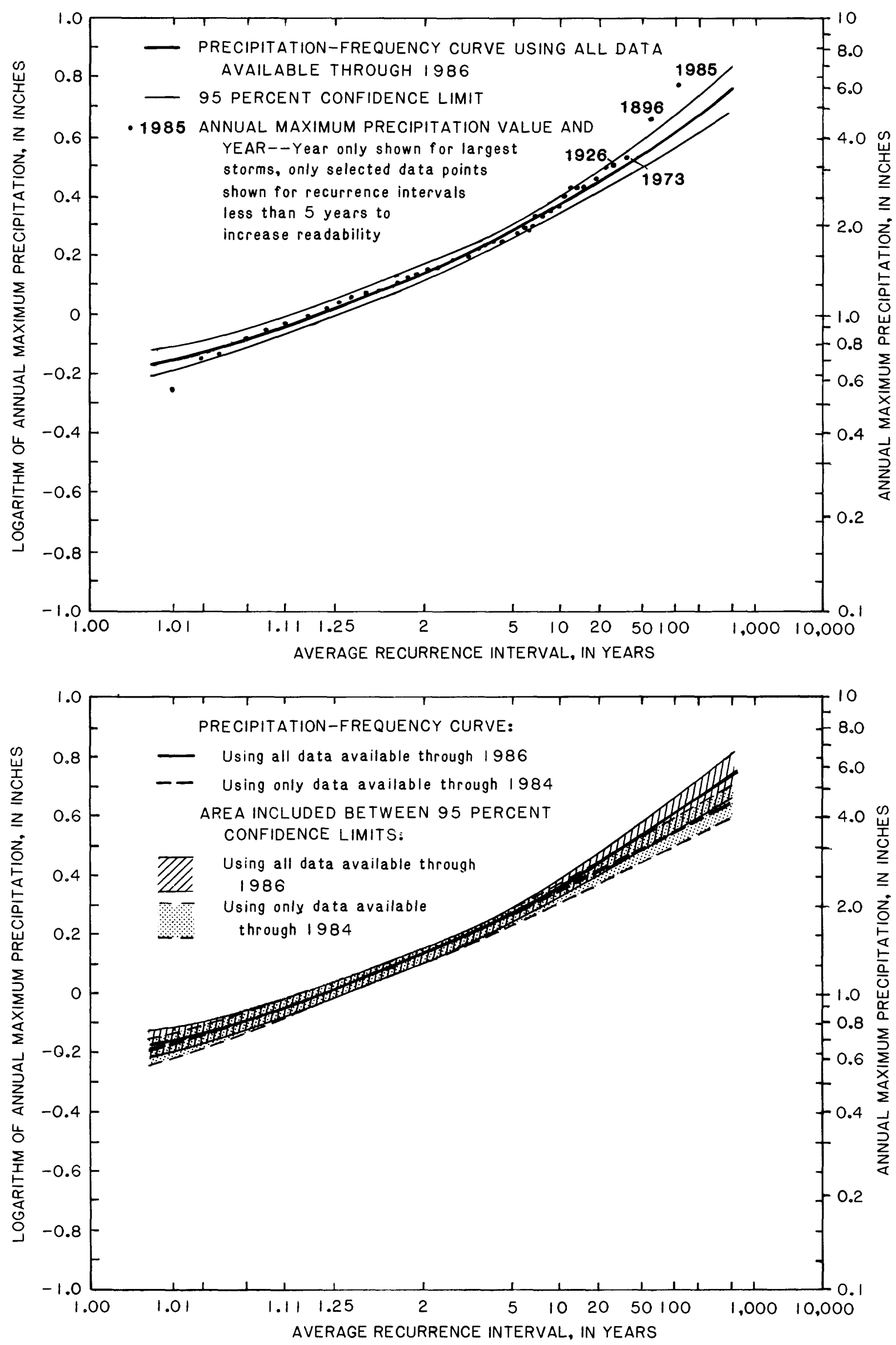

Figure 4g.--Precipitation-frequency curves for 24-hour duration, Pearson Type III distribution. 


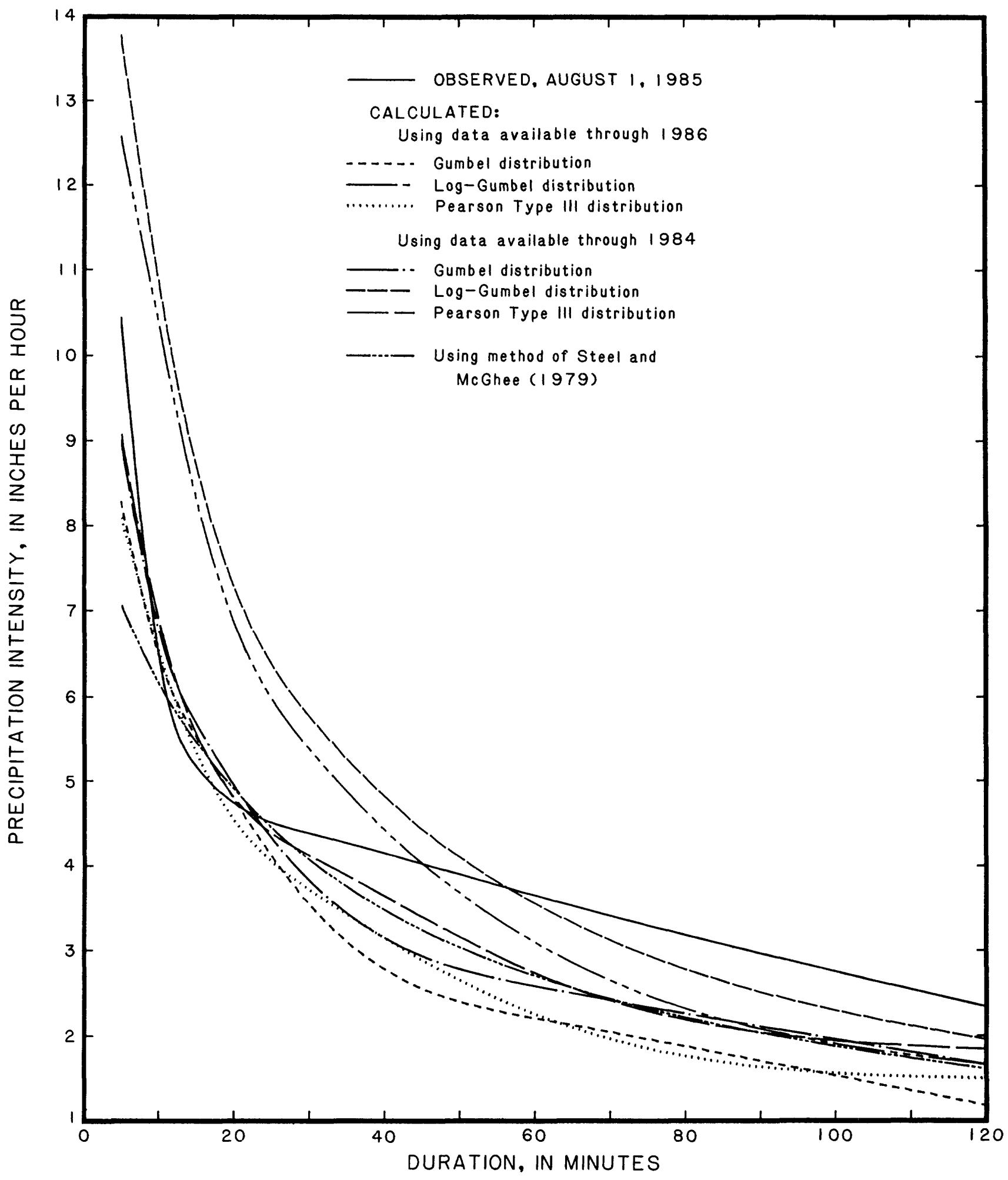

Figure 5.--Intensity-duration curves for 100-year storm. 
Calculated 100-year-storm intensity-duration curves based on the Gumbel, log-Gumbe1, and log-Pearson Type III distributions are also plotted on figure 5. For comparison purposes, the observed intensity-duration curve for the storm of August 1, 1985, is plotted on the same figure. The same data are presented in tabular form in table 3. Intensities for selected durations for storms of other recurrence intervals are presented in table 4 .

\section{Results}

The intensity-duration curves ( $f$ ig. 5) based only on data collected through 1984 approximately overlie each other, and, as a group, 1 ie below the curves based on data collected through 1986 and on the method of Steel and McGhee (1979). The curves representing the log-Gumbel distribution 1 ie above the other curves; however, the log-Gumbel distribution probably is overpredicting the intensity of precipitation because the skewness of the actual data (table 2) is different than the skewness of 1.14 assumed in the Gumbel distribution. Similarly, the arithmetic Gumbel distribution may be slightly underpredicting the intensity of precipitation. The log-Pearson Type III distribution may better fit this data because only the log-Pearson distribution incorporates the value of skewness of the observed data in calculating the fitted curve. Although the calculated value of skewness may be affected by sampling error, this effect should be minimized in the case of the Cheyenne data due to the long period of record.

The curve for the storm of August 1, 1985, 1ies above al1 the curves plotted on the figure for the 5-minute and 1-hour durations except those representing the log-Gumbel distributions, and the curve for the storm of August 1 ies above all the other curves for the 2-hour duration, indicating that this storm was unusually intense for these durations.

\section{HYETOGRAPH SHAPE AND PATTERN INDEX}

In order for runoff to occur, precipitation rate must exceed infiltration rate. Only the amount of precipitation in excess of the infiltration rate (infiltration rate is zero for fully saturated ground) is available to run off. If a period of intense precipitation occurs when the ground is dry (as at the beginning of a storm), some of the precipitation will infiltrate into the soil, and only the remainder will run off. However, when the ground is saturated, virtually all of the precipitation will be available to run off. If precipitation occurs when the ground is dry, both a smaller volume of runoff and a smaller peak flow will result than if precipitation of the same intensity and duration occurs when the ground is saturated.

Calculation of pattern index (Hasfurther and Tyrrel1, 1984) is one technique for examining storm hyetographs to determine whether the period of maximum precipitation intensity occurred early or late during the storm. Dimensionless hyetographs (cumulative precipitation versus time) are drawn for each storm, and the area under the curve is calculated either by graphical methods or numerical integration. This area, greater than zero but less than one, is the pattern index. A pattern index less than 0.5 indicates that the major part of the precipitation occurred after the 
Table 3.--Intensity-duration data for 100-year storm

\begin{tabular}{|c|c|c|c|c|c|c|}
\hline \multirow[b]{3}{*}{ Method of analysis } & \multicolumn{6}{|c|}{ Precipitation intensity, in inches per hour } \\
\hline & \multicolumn{6}{|c|}{ Duration } \\
\hline & $\begin{array}{l}5 \\
\text { min- } \\
\text { utes }\end{array}$ & $\begin{array}{l}10 \\
\text { min- } \\
\text { utes }\end{array}$ & $\begin{array}{l}15 \\
\text { min- } \\
\text { utes }\end{array}$ & $\begin{array}{l}30 \\
\text { min- } \\
\text { utes }\end{array}$ & $\begin{array}{c}1 \\
\text { hour }\end{array}$ & $\begin{array}{c}2 \\
\text { hours }\end{array}$ \\
\hline Observed, August 1, 1985 & $\begin{array}{r}10.44 \\
\text { Calculat } \\
\end{array}$ & $\begin{array}{r}6.48 \\
\text { ed us in }\end{array}$ & $\begin{array}{l}5.16 \\
\text { data } \\
\end{array}$ & $\begin{array}{r}4.38 \\
\text { availab } \\
\end{array}$ & $\begin{array}{r}3.64 \\
\text { ethr } \\
\end{array}$ & $\begin{array}{r}2.34 \\
\text { gh } 1984 \\
\end{array}$ \\
\hline $\begin{array}{l}\text { Gumbe } 1 \text { distribution } \\
\text { Log-Gumbe } 1 \text { distribution } \\
\text { Log-Pearson distribution }\end{array}$ & $\begin{array}{r}8.28 \\
12.57 \\
8.09\end{array}$ & $\begin{array}{r}6.48 \\
10.43 \\
6.53\end{array}$ & $\begin{array}{l}5.52 \\
8.36 \\
5.33\end{array}$ & $\begin{array}{l}3.54 \\
5.38 \\
3.71\end{array}$ & $\begin{array}{l}2.20 \\
3.09 \\
2.25\end{array}$ & $\begin{array}{l}1.18 \\
1.66 \\
1.50\end{array}$ \\
\hline & \multicolumn{6}{|c|}{ Calculated using data available through 1986} \\
\hline $\begin{array}{l}\text { Gumbe } 1 \text { distribution } \\
\text { Log-Gumbe } 1 \text { distribution } \\
\text { Log-Pearson distribution }\end{array}$ & $\begin{array}{r}9.00 \\
13.78 \\
9.07\end{array}$ & $\begin{array}{r}6.78 \\
10.92 \\
6.90\end{array}$ & $\begin{array}{l}5.68 \\
8.75 \\
5.56\end{array}$ & $\begin{array}{l}3.82 \\
5.77 \\
4.12\end{array}$ & $\begin{array}{l}2.58 \\
3.55 \\
2.73\end{array}$ & $\begin{array}{l}1.66 \\
1.95 \\
1.84\end{array}$ \\
\hline & \multicolumn{6}{|c|}{ Calculated using regional equations } \\
\hline Method of Steel and McGhee & 7.06 & 6.16 & 5.46 & 4.07 & 2.70 & 1.61 \\
\hline
\end{tabular}

midpoint of the storm. Conversely, a pattern index greater than 0.5 indicates that the major part of the precipitation occurred before the midpoint of the storm.

Pattern index is not the only factor that can affect the amount of runoff produced by a given storm. Amount of moisture already in the soil from antecedant precipitation or irrigation can affect the amount of infiltration, and hence the amount of water available to run off. Channelization by streets and gutters, paving of open space, and other effects of urbanization can have a major impact on quantity of runoff by reducing either infiltration, or depression storage, or both. Infiltration, however, has little impact on quantity of runoff in areas of lowpermeability soil or sloping terrain for storms of more than about a 20-year recurrence interval (A. M. Wacker, Wyoming State Highway Department, written commun., 1987). Because of all these effects on quantity of infiltration, the recurrence interval of runoff will rarely be the same as the recurrence interval of the storm that caused the runoff. 
Table 4.--Precipitation intensity for storms of selected recurrence

intervals and durations, using data available through 1986

\begin{tabular}{|c|c|c|c|c|c|c|}
\hline \multirow[b]{3}{*}{ Duration } & \multicolumn{6}{|c|}{ Precipitation intensity, in inches per hour } \\
\hline & \multicolumn{6}{|c|}{ Recurrence interval, in years } \\
\hline & 2 & 5 & 10 & 25 & 50 & 100 \\
\hline & \multicolumn{6}{|c|}{ Gumbel distribution } \\
\hline 5 minutes & 3.48 & 4.92 & 5.88 & 7.20 & 8.04 & 9.00 \\
\hline 10 minutes & 2.58 & 3.72 & 4.44 & 5.40 & 6.06 & 6.78 \\
\hline 15 minutes & 2.20 & 3.13 & 3.76 & 4.52 & 5.12 & 5.68 \\
\hline 30 minutes & 1.34 & 2.00 & 2.44 & 3.00 & 3.42 & 3.82 \\
\hline 1 hour & .79 & 1.27 & 1.59 & 1.99 & 2.28 & 2.58 \\
\hline 2 hours & .46 & .78 & 1.00 & 1.26 & 1.46 & 1.66 \\
\hline \multirow[t]{2}{*}{24 hours } & .06 & .09 & .11 & .13 & .15 & .17 \\
\hline & \multicolumn{6}{|c|}{ Log-Gumbel distribution } \\
\hline 5 minutes & 3.23 & 4.67 & 6.15 & 8.49 & 10.70 & 13.78 \\
\hline 10 minutes & 2.44 & 3.62 & 4.77 & 6.58 & 8.47 & 10.92 \\
\hline 15 minutes & 2.00 & 2.97 & 3.82 & 5.39 & 6.79 & 8.75 \\
\hline 30 minutes & 1.26 & 1.91 & 2.46 & 3.48 & 4.48 & 5.77 \\
\hline 1 hour & .74 & 1.12 & 1.48 & 2.09 & 2.75 & 3.55 \\
\hline 2 hours & .41 & .62 & .83 & 1.17 & 1.51 & 1.95 \\
\hline \multirow[t]{2}{*}{24 hours } & .05 & .08 & .10 & .13 & .16 & .20 \\
\hline & \multicolumn{6}{|c|}{ Log-Pearson Type III distribution } \\
\hline 5 minutes & 3.42 & 4.75 & 5.70 & 6.98 & 8.00 & 9.07 \\
\hline 10 minutes & 2.64 & 3.66 & 4.38 & 5.40 & 6.12 & 6.90 \\
\hline 15 minutes & 2.20 & 3.04 & 3.60 & 4.36 & 4.96 & 5.56 \\
\hline 30 minutes & 1.34 & 1.96 & 2.42 & 3.06 & 3.56 & 4.12 \\
\hline 1 hour & .73 & 1.10 & 1.41 & 1.87 & 2.27 & 2.73 \\
\hline 2 hours & .41 & .63 & .83 & 1.16 & 1.46 & 1.84 \\
\hline 24 hours & .06 & .08 & .10 & .13 & .15 & .18 \\
\hline
\end{tabular}

\section{Method of Analys is}

Dimensionless hyetographs of cumulative precipitation versus time were drawn for nine flood-producing storms that occurred between 1904 and 1973; total precipitation for these storms ranged from 0.57 to 2.70 inches. The hyetographs were based on the 5-minute incremental precipitation data of Craig and Rankl (1978). In addition, a dimensionless hyetograph was drawn for the storm of August 1, 1985. Pattern index was calculated for each of the storms by graphical methods. The August 1 storm was compared to the other storms to determine whether it was anomalous. 


\section{Results}

Dimensionless hyetographs for nine storms identified as major floodproducing storms in Druse and others (1986) and the storm of August 1, 1985, are plotted in figure 6. The 10-percent and 90-percent 1 imit curves for thunderstorms in Wyoming, as described by Hasfurther and Tyrrel1 (1984) are plotted on the same figure. Pattern indicies were calculated for the 10 storms and are presented in table 5 .

The dimensionless hyetograph for the August 1 storm 1 ies below the other curves in figure 6, and the pattern index for the August 1 storm is smaller than the pattern index for each of the other nine storms (table 2). The mean pattern index for the nine storms from 1904 through 1973 is 0.63 with a standard deviation of 0.064 . The storm of August 1 has a pattern index of only 0.40, three standard deviations below the mean, and is an outlier. The August 1 storm started with a smaller dimensionless rainfall intensity, and increased to greater intensity during the latter part of the storm, than did other historical flood-producing storms for which records were available. A storm having these characteristics would tend to have a larger peak runoff than a storm of equal magnitude, duration, and maximum precipitation intensity but higher pattern index.

Table 5.--Storm characteristics for 10 flood-producing storms

\begin{tabular}{lccc}
\hline $\begin{array}{c}\text { Date of } \\
\text { storm }\end{array}$ & $\begin{array}{c}\text { Length of } \\
\text { storm } \\
\text { (minutes) }\end{array}$ & $\begin{array}{c}\text { Total precipi- } \\
\text { tation } \\
\text { (inches) }\end{array}$ & $\begin{array}{c}\text { Pattern } \\
\text { index } \\
\text { (dimensionless) }\end{array}$ \\
\hline May 20, 1904 & 105 & 1.05 & 0.67 \\
July 18, 1918 & 50 & .80 & .76 \\
August 14, 1918 & 30 & .57 & .61 \\
June 14, 1926 & 60 & 2.50 & .65 \\
June 10-11, 1935 & 25 & 1.30 & .64 \\
August 26, 1946 & 35 & 1.45 & .65 \\
June 14, 1955 & 265 & 2.40 & .60 \\
Ju1y 19, 1973 & 775 & 3.27 & .52 \\
September 8, 1973 & 85 & 2.70 & .57 \\
August 1, 1985 & 210 & 6.06 & .40 \\
\hline
\end{tabular}




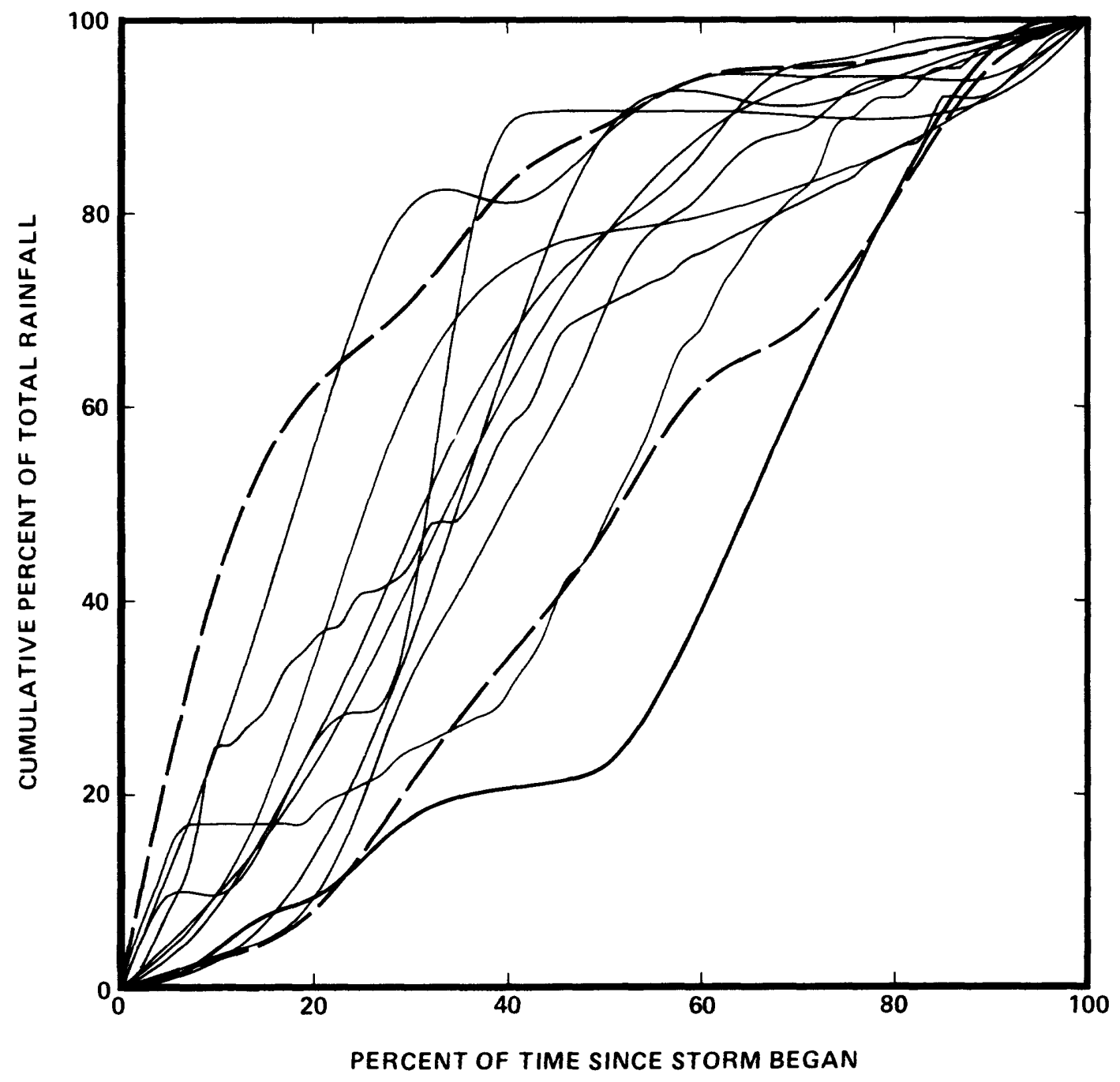

Figure 6.--Dimensionless hyetographs for 10 flood-producing storms. 
Annual maximum precipitation data for Cheyenne, Wyoming, have been collected for durations of $5,10,15$, and 30 minutes, and 1 and 2 hours since 1902 and continuing to the present. Annual maximum precipitation data for 24-hour duration have been collected beginning as early as 1871. Data were plotted and analyzed using three different assumptions: (1) The data could be described by an arithmetic Gumbel distribution; (2) the logarithms of the data could be described by a Gumbel distribution; and ( 3 ) the logarithms of the data could be described by a Pearson Type III distribution. For each of these distributions, the data were examined twice. First, the "previously defined" precipitation-freqency curve was developed, using data collected before 1985. Updated curves were then developed, using all data collected through 1986, in order to examine the effect that the large thunderstorm of August 1, 1985, had on the predicted magnitude of storms of long recurrence interval. The inclusion of the storm of August 1, 1985, had the most noticeable effect on the prediction of the amount of precipitation expected in the 1 - and 2-hour durations, and the least noticeable effect on the prediction of the amount of precipitation expected in the $10-$ and 15-minute durations, regardless of which distribution was assumed to describe the data. The predicted magnitude of storms was similar (within 10 percent) for all distributions for storms of less than 25-year recurrence interval. Predicted magnitude of storms with extremely long recurrence intervals varied greatly depending on which distribution was assumed to describe the data. The skewness both of the arithmetic and of the logarithmically transformed data was different than the value of 1.14 assumed in the Gumbel distribution, which caused the Gumbel distribution to underpredict--and the log-Gumbel distribution to overpredict--the amount of precipitation expected for a given recurrence interval. Therefore, the log-Pearson Type III distribution was considered the most suitable for describing the Cheyenne precipitation data.

Four different methods of analysis were used to develop seven intensity-duration curves for the 100-year storm. These curves were compared to the observed curve for the August 1, 1985, storm. For durations greater than 30 minutes, the observed curve indicates greater intensity than do five of the seven calculated curves.

Dimensionless hyetographs were developed for 10 flood-producing storms that have occurred in the Cheyenne area since 1903. Pattern index (integral of the dimensionless hyetograph curve) was calculated, and the index for the storm of August 1, 1985, is three standard deviations lower than the mean of the pattern indices for the other nine storms, indicating that the distribution of precipitation over time for the August 1 storm was outside the normal range for Cheyenne. 
Chow, V.T., ed., 1964, Handbook of applied hydrology: New York, McGrawHil1, p. 8-1 to 8-35.

Craig, G.S., and Rank1, J.G., 1978, Analysis of runoff from small drainage basins in Wyoming: U.S. Geological Survey Water-Supply Paper 2056, $70 \mathrm{p}$.

Druse, S.A., Cooley, M.E., Green, S.L., and Lowham, H.W., 1986, Flood of August 1, 1985, in Cheyenne, Wyoming: U.S. Geological Survey Hydrologic Investigations Atlas HA-699, scales 1:6,000 and 1:48,000, two sheets.

Hasfurther, V.R., and Tyrrell, P.T., 1984, Semi-arid storm hyetograph properties in Wyoming: Transportation Research Record 995, p. 50-59.

Interagency Advisory Committee on Water Data, 1982, Guidelines for determining flood flow freqency: Hydrology Subcommittee Bulletin 17B, $180 \mathrm{p}$.

Lepkin, W.D., DeLapp, M.M., Kirby, W.H., and Wilson, T.A., 1979, Annua1 flood frequency analysis using U.S. Water Resources Council Guidelines (J407), WATSTORE Users Guide: U.S. Geological Survey Open-File Report 79-1336-I, v. 4, Chapter 1-C.

Morel-Seytoux, H.J., 1986, Eng ineering hydrology--ensemble of lecture notes and class handouts developed since 1977: Fort Collins, Hydrowar Program, CER85-86HJM15, Colorado State University, 528 p.

National Oceanic and Atmospheric Administration, Environmental Data, Satellite and Information Service, 1985-86, Local climatological datamonthly summary, Wyoming, 1985-86: Asheville, North Carolina.

Stee1, E.W., and McGhee, T.J., 1979, water supply and sewerage (5th ed.): New York, McGraw Hill, 665 p.

U.S. Army Corps of Engineers, 1969, Flood plain information, Dry Creek, v. I, Cheyenne, Wyoming: Omaha, Nebraska, 23 p. 